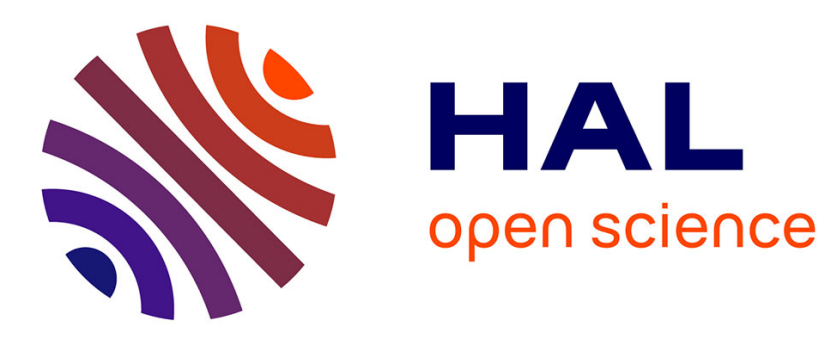

\title{
Coordination chemistry of diphenylphosphinoferrocenylthioethers on cyclooctadiene and norbornadiene rhodium(i) platforms
}

Ekaterina Kozinets, Oleksandr Koniev, Oleg Filippov, Jean-Claude Daran, Rinaldo Poli, Elena V Shubina, Natalia V Belkova, Eric Manoury

\section{To cite this version:}

Ekaterina Kozinets, Oleksandr Koniev, Oleg Filippov, Jean-Claude Daran, Rinaldo Poli, et al.. Coordination chemistry of diphenylphosphinoferrocenylthioethers on cyclooctadiene and norbornadiene rhodium(i) platforms. Dalton Transactions, 2012, 41 (38), pp.11849. 10.1039/c2dt30993a . hal02909996

\section{HAL Id: hal-02909996 https://hal.science/hal-02909996}

Submitted on 3 Mar 2021

HAL is a multi-disciplinary open access archive for the deposit and dissemination of scientific research documents, whether they are published or not. The documents may come from teaching and research institutions in France or abroad, or from public or private research centers.
L'archive ouverte pluridisciplinaire HAL, est destinée au dépôt et à la diffusion de documents scientifiques de niveau recherche, publiés ou non, émanant des établissements d'enseignement et de recherche français ou étrangers, des laboratoires publics ou privés. 


\title{
Coordination chemistry of diphenylphosphinoferrocenylthioethers on cyclooctadiene and norbornadienerhodium(I) platforms
}

\author{
Ekaterina M. Kozinets, ${ }^{a, b, c}$ Oleksandr Koniev ${ }^{a, b}$ Oleg A. Filippov, ${ }^{c}$ Jean-Claude Daran, ${ }^{a, b}$ Rinaldo \\ Poli, ${ }^{a, b, d}$ Elena S. Shubina, ${ }^{c}$ Natalia V. Belkova, ${ }^{* c}$ and Eric Manoury $* a, b$
}

\author{
${ }_{5}$ Received (in $\left.X X X, X X X\right)$ Xth $X X X X X X X X X 20 X X$, Accepted Xth XXXXXXXXX 20XX \\ DOI: $10.1039 / b 000000 x$
}

Complexes [ $\mathrm{RhCl}($ diene $)(\mathrm{P}, \mathrm{SR})]$ with chiral ferrocenyl phosphine-thioethers ligands (diene $=$ norbornadiene, NBD, $\mathbf{1}^{\mathbf{R}}$, or 1,5-cyclooctadiene, $\mathrm{COD}, \mathbf{3}^{\mathbf{R}} ; \mathrm{P}, \mathrm{SR}=\mathrm{CpFe}\left(1,2-\eta^{5}-\mathrm{C}_{5} \mathrm{H}_{3}\left(\mathrm{PPh}_{2}\right)\left(\mathrm{CH}_{2} \mathrm{SR}\right) ; \mathrm{R}=\right.$ $t \mathrm{Bu}, \mathrm{Ph}, \mathrm{Bz}, \mathrm{Et})$ and the corresponding $[\mathrm{Rh}($ diene $)(\mathrm{P}, \mathrm{SR})]\left[\mathrm{BF}_{4}\right]\left(\right.$ diene $\left.=\mathrm{NBD}, \mathbf{2}^{\mathrm{R}} ; \mathrm{COD}, \mathbf{4}^{\mathbf{R}}\right)$ have been

10 synthesized from $[\mathrm{RhCl}(\text { diene })]_{2}$ and the appropriate $\mathrm{P}, \mathrm{SR}$ ligand. The molecular structure of the cationic complexes $2^{t \mathrm{Bu}}, \mathbf{4}^{\mathrm{Ph}}$ and $\mathbf{4}^{\mathrm{Bz}}$, determined by single-crystal X-ray diffraction, shows the expected slightly distorded square planar geometry. For the neutral chloride complexes, a combination of experimental IR and computational DFT investigations points to an equally four coordinate square planar geometry with the diene ligand, the chlorine and the phosphorus atoms in the coordination sphere and with a dangling

15 thioether function. However, a second isomeric form featuring a 5-coordinated square planar geometry with the thioether function placed in the axial position is easily accessible in some cases.

\section{Introduction}

Since the development by Noyori and coworkers of efficient catalytic systems for the asymmetric hydrogenation of non func20 tionalized ketones ${ }^{1-3}$, much effort has been devoted to the asymmetric hydrogenation of polar substrates, ${ }^{4}$ ketones ${ }^{5}$ but also imines $^{6-9}$ or heteroarenes, ${ }^{10}$ because of their great scientific and practical importance, which includes industrial applications. ${ }^{11,12}$ Most of the catalysts used so far are based on ruthenium, rhodium 25 and iridium with phosphorus and nitrogen-containing ligands.

Our group has worked extensively on the chiral ligand platform built around the planar chirality of ferrocene, mostly with asymmetric 1,2 substitution, but also 1,2,3 trisubstitution and 1,2,3,1' tetrasubstitution. ${ }^{13-17}$ Our interest in recent years has 30 focused on chiral ligands based on $\mathrm{P}$ and $\mathrm{S}$ donor atoms with the development of the 1,2-substituted ferrocenes (P,SR) shown in Scheme 1. ${ }^{18-22}$ The use of these ligands (particularly for $\mathrm{R}=\mathrm{Et}$, $\mathrm{Ph}, \mathrm{Bz}$ and $t \mathrm{Bu}$ ) has led to a number of catalytic applications in asymmetric allylic substitution ${ }^{23,24}$ asymmetric methoxycarbo35 nylation ${ }^{25}$ and asymmetric hydrogenation. ${ }^{26}$ Although other kinds of ligands based on $\mathrm{P}$ and $\mathrm{S}$ donor atoms have already been used in $\mathrm{C}=\mathrm{C}$ hydrogenations, ${ }^{27-30}$ we reported the first application of these types of ligands, to the best of our knowledge, to the hydrogenation of ketones, promising results being obtained in the 40 ionic hydrogenation of various acetophenones. ${ }^{26}$

In combination with $[\operatorname{IrCl}(\mathrm{COD})]_{2} \quad(\mathrm{COD}=1,5$-cyclooctadiene), these ligands have yielded the corresponding $\mathrm{IrCl}(\mathrm{COD})(\mathrm{P}, \mathrm{SR})$ adducts, of which those with $\mathrm{R}=\mathrm{Et}, \mathrm{Ph}$ and $\mathrm{Bz}$ adopt a 5-coordinate square pyramidal geometry (axial $\mathrm{Cl}$ ) in the 45 solid state and in solution, whereas the $t \mathrm{Bu}$ derivative is square planar with a dangling thioether function. ${ }^{31}$ These iridium complexes were shown to be excellent precatalysts for the hydrogenation of aromatic ketones in the presence of a strong base such as NaOMe in methanol and other alcohols, leading to 50 quantitative hydrogenations in less than two hours at room temperatures under 30 bar of $\mathrm{H}_{2}$ and with only $0.05 \%$ of catalyst loading and with enantiomeric excesses that reached $>99 \%$ (at $0^{\circ} \mathrm{C}$ ) for selected substrates. ${ }^{26}$

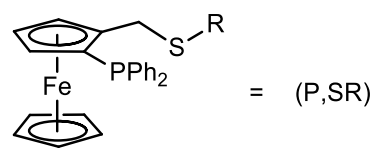

Scheme 1. General structure of the (P,SR) ligands used in this study.

Specific stoichiometric investigations of the precatalyst hydrogenation in methanol, yielding partial hydrogenation of the cyclooctadiene to cyclooctene (experimentally observed by GC${ }_{60} \mathrm{MS}$ ) and presumably leading at least initially to $\operatorname{Ir}(\mathrm{P}, \mathrm{SR})(\mathrm{OMe})(\mathrm{MeOH})$, have not so far allowed the isolation or spectroscopic observation of a catalytically active species. It is known from elegant work by Heller et al. that the hydrogenation of diene ligands in complexes such as $\left[\mathrm{M}(\text { diene })\left(\mathrm{L}_{2}\right)\right]^{+}\left(\mathrm{L}_{2}\right.$ being ${ }_{65}$ typically a diphosphine ligand a $\mathrm{M}=\mathrm{Rh}$ ) in methanol yields $\left[\mathrm{M}\left(\mathrm{L}_{2}\right)(\mathrm{MeOH})_{2}\right]^{+}$adducts and that such derivatives are less easily generated and less stable when $\mathrm{M}=\mathrm{Ir} .{ }^{32-35}$ With the goal of generating more stable model complexes of the above mentioned iridium catalysts, we have therefore turned out attention to 70 analogous $\mathrm{Rh}$ complexes. We report in this contribution the synthesis and characterization of a variety of adducts of the $(\mathrm{P}, \mathrm{SR})$ ligands in Scheme 1 with $[\mathrm{RhCl}(\mathrm{COD})]_{2}$ and $[\mathrm{RhCl}(\mathrm{NBD})]_{2} \quad(\mathrm{NBD}=$ norbornadiene). This includes an IR study and a DFT investigation to elucidate the structure of the 75 compound in the solid state and in solution. Related cationic complexes $[\mathrm{Rh}(\mathrm{COD})(\mathrm{P}, \mathrm{SR})]^{+}$and $[\mathrm{Rh}(\mathrm{NBD})(\mathrm{P}, \mathrm{SR})]^{+}$have also been isolated and structurally characterized. We also report preliminary investigations of acetophenone hydrogenation using these rhodium complexes as precatalysts. It will be shown that, 80 albeit the catalytic activity is lower, these complexes are good structural and functional models of the corresponding Ir precatalysts.

\section{Experimental Section}


General. All reactions were carried out under an argon atmosphere using standard Schlenk techniques. Solvents were carefully dried by conventional methods and distilled under argon before use. The (R/S)-2-diphenylphosphanyl-(R-thiomethyl)5 ferrocene ligands $(\mathrm{R}=\mathrm{Et}, t \mathrm{Bu}, \mathrm{Ph}, \mathrm{Bz})$ were prepared according to a published procedure from racemic 2-(diphenylthiophosphanylferrocenyl)methanol. ${ }^{36}$ Compounds $[\mathrm{RhCl}(\mathrm{COD})]_{2}$, $[\mathrm{RhCl}(\mathrm{NBD})]_{2}$ and $\left[\mathrm{Rh}(\mathrm{COD})_{2}\right] \mathrm{BF}_{4}$ were purchased from Strem Chemicals and used as received. ${ }^{1} \mathrm{H},{ }^{13} \mathrm{C}\left\{{ }^{1} \mathrm{H}\right\}$ and ${ }^{31} \mathrm{P}\left\{{ }^{1} \mathrm{H}\right\}$ NMR 10 spectra were recorded with a Bruker Avance 500 FT-NMR spectrometer. The resonances were calibrated relative to the residual solvent peaks and are reported with positive values downfield from TMS. For all characterized compounds, the peak assignments in the ${ }^{1} \mathrm{H}$ and ${ }^{13} \mathrm{C}$ NMR spectra were based on ${ }_{15}$ COSY, HSQC and HMBC 2D experiments. HRMS were obtained from dichloromethane solutions with a Xevo G2 Q TOF spectrometer by the electrospray method. IR spectra were recorded at room temperature with a Nicolet 6700 spectrometer in the solid state (as nujol mulls) using polyethylene cells for the 20 low frequency region. The optical purity and the conversions for the hydrogenation experiments were determined by chiral GC (Supelco BETA DEX ${ }^{\mathrm{TM}}$ 225).

General procedure for the synthesis of $\mathrm{RhCl}(\mathrm{NBD})(\mathrm{P}, \mathrm{SR})$, 1. In a Schlenk tube, under nitrogen, ligand (P,SR) $(0.793 \mathrm{mmol})$ 25 was dissolved in dichloromethane $(5 \mathrm{~mL})$ and $[\mathrm{RhCl}(\mathrm{NBD})]_{2}$ (183 mg, $0.396 \mathrm{mmol}$ ) was added. The solution was stirred for 4 $\mathrm{h}$ at room temperature and $15 \mathrm{~mL}$ of pentane was then added to yield a yellow precipitate. The precipitate was filtered under argon and washed with pentane to give $\mathrm{RhCl}(\mathrm{NBD})(\mathrm{P}, \mathrm{SR})$.

${ }_{30} \operatorname{RhCl}(\mathbf{N B D})(\mathbf{P}, \mathbf{S t B u})\left(\mathbf{1}^{\mathbf{t B u}}\right.$, yield: $\left.97 \%\right) .{ }^{1} \mathrm{H}$ NMR $(500 \mathrm{MHz}$, $\left.\mathrm{CDCl}_{3}\right): \delta 8.48(\mathrm{~m}, 2 \mathrm{H}, \mathrm{Ph}) ; 7.53(\mathrm{~m}, 3 \mathrm{H}, \mathrm{Ph}) ; 7.3-7.2(\mathrm{~m}, 3 \mathrm{H}$, $\mathrm{Ph}) ; 6.93$ (brdd, $\left.2 \mathrm{H}, \mathrm{Ph}, \mathrm{J}_{\mathrm{HH}}=7.2 \mathrm{~Hz}, \mathrm{~J}_{\mathrm{HP}}=9.7 \mathrm{~Hz}\right) ; 5.06(\mathrm{~d}$ (AB), $1 \mathrm{H}, \underline{\mathrm{C}}_{2} \mathrm{Fc}, \mathrm{J}_{\mathrm{HH}}=13.8 \mathrm{~Hz}$ ); 4.56 (s, 1H, subst. Cp); 4.30 (s, $1 \mathrm{H}$, subst. Cp); 4.14 (s, 1H, subst. Cp); 3.90 (s, 5H, Cp); 3.84 (d $\left.35(\mathrm{AB}), 1 \mathrm{H}, \mathrm{C}_{2} \mathrm{Fc}, \mathrm{J}_{\mathrm{HH}}=13.8 \mathrm{~Hz}\right) ; 3.78$ (s, 2H, CH NBD); 3.61 (s, 2H, CH NBD); 3.57 (s, 2H, CH NBD); 1.51 (s, 9H, tBu) 1.23 (s, $\left.2 \mathrm{H}, \mathrm{CH}_{2} \mathrm{NBD}\right) .{ }^{13} \mathrm{C}\left\{{ }^{1} \mathrm{H}\right\} \mathrm{NMR}\left(125 \mathrm{MHz}, \mathrm{CDCl}_{3}\right): \delta 136.3(\mathrm{~d}$, $\left.\mathrm{Ph}, \mathrm{J}_{\mathrm{CP}}=12.8 \mathrm{~Hz}\right) ; 135.3\left(\mathrm{~d}\right.$, quatPh, $\left.\mathrm{J}_{\mathrm{CP}}=45.6 \mathrm{~Hz}\right) ; 133.8(\mathrm{~d}$, quatPh, $\left.\mathrm{J}_{\mathrm{CP}}=45.6 \mathrm{~Hz}\right) ; 132.3\left(\mathrm{~d}, \mathrm{Ph}, \mathrm{J}_{\mathrm{CP}}=9.2 \mathrm{~Hz}\right) ; 130.4(\mathrm{~d}, \mathrm{Ph}$, $\left.{ }_{40} \mathrm{~J}_{\mathrm{CP}}=2.2 \mathrm{~Hz}\right) ; 128.7\left(\mathrm{~d}, \mathrm{Ph}, \mathrm{J}_{\mathrm{CP}}=1.8 \mathrm{~Hz}\right) ; 127.7\left(\mathrm{~d}, \mathrm{Ph}, \mathrm{J}_{\mathrm{CP}}=10.4\right.$ $\mathrm{Hz}) ; 127.2\left(\mathrm{~d}, \mathrm{Ph}, \mathrm{J}_{\mathrm{CP}}=9.2 \mathrm{~Hz}\right) ; 91.3\left(\mathrm{~d}\right.$, quatCp, $\left.\mathrm{J}_{\mathrm{CP}}=18.6 \mathrm{~Hz}\right)$; 73.3(s, substCp); 71.9 (d, substCp, $\mathrm{J}_{\mathrm{CP}}=7.6 \mathrm{~Hz}$ ); 71.5 (d, quatCp, $\mathrm{J}_{\mathrm{CP}}=7.6 \mathrm{~Hz}$ ); 71.0 (s, Cp); 69.0 (d, substCp, $\mathrm{J}_{\mathrm{CP}}=5.2 \mathrm{~Hz}$ ); 61.4 (s, $\mathrm{CH}_{2}$ NBD); 60.2(br s, CH NBD); 55.9 (br s, CH NBD); 48.5 45 (s, CH NBD); 46.0(s, S- $\left.\underline{\mathrm{C}}\left(\mathrm{CH}_{3}\right)\right) ; 31.3$ (s, $\left.\mathrm{S}-\mathrm{C}\left(\underline{\mathrm{CH}}_{3}\right)\right) ; 28.8$ $\left(\mathrm{CH}_{2} \mathrm{Fc}\right) .{ }^{31} \mathrm{P}\left\{{ }^{1} \mathrm{H}\right\}$ NMR $\left(202 \mathrm{MHz}, \mathrm{CDCl}_{3}\right): \delta 23.6(\mathrm{~d}, \mathrm{JP}-\mathrm{Rh}=$

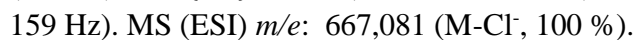

RhCl(NBD)(P,SPh) $\left(\mathbf{1}^{\mathbf{P h}}\right.$,yield: $\left.98 \%\right) .{ }^{1} \mathrm{H}$ NMR (500 MHz, $\left.\mathrm{CDCl}_{3}\right): \delta 8.52(\mathrm{~m}, 2 \mathrm{H}, \mathrm{Ph}) ; 7.73\left(\right.$ br d, $\mathrm{J}_{\mathrm{HH}}=7.5 \mathrm{~Hz}, 2 \mathrm{H}, \mathrm{Ph}$ ); ${ }_{50} 7.58$ (m, 3H, Ph); 7.4-7.1 (m, 3H, Ph); 6.62 (m, 2H, Ph); 5.37 (br $\mathrm{d}(\mathrm{AB}), 1 \mathrm{H}, \underline{\mathrm{C}}_{2} \mathrm{Fc}, \mathrm{J}_{\mathrm{HH}}=13 \mathrm{~Hz}$ ); 4.45 (br s, 1H, subst. Cp); 4.28 (br d (AB), $1 \mathrm{H}, \mathrm{C}_{2} \mathrm{Fc}, \mathrm{J}_{\mathrm{HH}}=13 \mathrm{~Hz}$ ); 4.24 (br s, 1H, subst. Cp); 4.03 (br s, $1 \mathrm{H}$, subst. Cp); 3.88 (s, 5H, Cp); 3.54 (s, 4H, CH $\mathrm{NBD}) ; 3.40$ (s, 2H, CH NBD); 1.17 (s, 2H, CH $2 \mathrm{NBD}) .{ }^{13} \mathrm{C}\left\{{ }^{1} \mathrm{H}\right\}$ ${ }_{55} \mathrm{NMR}\left(125 \mathrm{MHz}, \mathrm{CDCl}_{3}\right): \delta 136.2\left(\mathrm{~d}, \mathrm{Ph}, \mathrm{J}_{\mathrm{CP}}=13.0 \mathrm{~Hz}\right) ; 135.3$ (d, quat $\mathrm{Ph}, \mathrm{J}_{\mathrm{CP}}=46.8 \mathrm{~Hz}$ ); 135.1 (quat $\mathrm{Ph}$ ); 133.3 (d, quat $\mathrm{Ph}, \mathrm{J}_{\mathrm{CP}}$ $=46.0 \mathrm{~Hz}) ; 131.6\left(\mathrm{~d}, \mathrm{Ph}, \mathrm{J}_{\mathrm{CP}}=9.3 \mathrm{~Hz}\right) ; 130.6\left(\mathrm{~d}, \mathrm{Ph}, \mathrm{J}_{\mathrm{CP}}=2.2\right.$ $\mathrm{Hz}) ; 130.3(\mathrm{Ph}) ; 129.0(\mathrm{Ph}) ; 128.6$ (d, Ph, J $\mathrm{CP}=1.8 \mathrm{~Hz}) ; 127.9$ (d,
$\left.\mathrm{Ph}, \mathrm{J}_{\mathrm{CP}}=10.5 \mathrm{~Hz}\right) ; 127.5(\mathrm{Ph}) ; 127.2\left(\mathrm{~d}, \mathrm{Ph}, \mathrm{J}_{\mathrm{CP}}=9.4 \mathrm{~Hz}\right) ; 89.0$ ${ }_{60}\left(\mathrm{~d}\right.$, quat $\left.\mathrm{Cp}, \mathrm{J}_{\mathrm{CP}}=18.6 \mathrm{~Hz}\right) ; 72.6$ (s, subst $\mathrm{Cp}$ ); 72.4 (d, subst Cp, $\left.\mathrm{J}_{\mathrm{CP}}=7.7 \mathrm{~Hz}\right) ; 71.0(\mathrm{~s}, \mathrm{Cp}) ; 70.8\left(\mathrm{~d}\right.$, quat $\left.\mathrm{Cp}, \mathrm{J}_{\mathrm{CP}}=40 \mathrm{~Hz}\right) ; 69.1$ $\left(\mathrm{d}, \mathrm{Ph}, \mathrm{J}_{\mathrm{CP}}=5.0 \mathrm{~Hz}\right) ; 60.8$ (d, $\left.\mathrm{J}_{\mathrm{CRh}}=5.3 \mathrm{~Hz}, \mathrm{CH}_{2} \mathrm{NBD}\right) ; 59.1(\mathrm{~d}$, $\mathrm{J}_{\mathrm{CRh}}=7.6 \mathrm{~Hz}, \mathrm{CH} \mathrm{NBD}$ ); 54.2 (br s, CH NBD); 48.2 (s, CH $\mathrm{NBD}) ; 34.1\left(\underline{\mathrm{CH}_{2} \mathrm{Fc}}\right) .{ }^{31} \mathrm{P}\left\{{ }^{1} \mathrm{H}\right\} \mathrm{NMR}\left(202 \mathrm{MHz}, \mathrm{CDCl}_{3}\right): \delta 23.8$ $65\left(\mathrm{~d}, \mathrm{~J}_{\mathrm{P}-\mathrm{Rh}}=154 \mathrm{~Hz}\right)$. MS (ESI) m/e: 687,049 (M-Cl- $\left.100 \%\right)$.

RhCl(NBD)(P,SBz) $\left(\mathbf{1}^{\mathrm{Bz}}\right.$,yield: 99\%). ${ }^{1} \mathrm{H}$ NMR (500 MHz, $\left.\mathrm{CDCl}_{3}\right): \delta 8.46(\mathrm{~m}, 2 \mathrm{H}, \mathrm{Ph}) ; 7.59(\mathrm{~m}, 3 \mathrm{H}, \mathrm{Ph}) ; 7.45-7.25(\mathrm{~m}, 8 \mathrm{H}$, $\mathrm{Ph}$ ); 6.88 (m, 2H, Ph); 4.32 (br s, 1H, subst. Cp); 4.25 (br s, $1 \mathrm{H}$, subst. Cp); 4.13 (br d (AB), $1 \mathrm{H}, \mathrm{CH}_{2} \mathrm{Fc}, \mathrm{J}_{\mathrm{HH}}=12 \mathrm{~Hz}$ ); 4.08 (s, $701 \mathrm{H}$, subst. $\mathrm{Cp}$ ); 4.00 (m, $\left.1 \mathrm{H} \mathrm{C} \underline{\mathrm{H}}_{2} \mathrm{Fc}+1 \mathrm{H} \mathrm{C} \underline{H}_{2} \mathrm{Ph}\right) ; 3.73$ (s, 5H, $\mathrm{Cp}$ ); 3.70 (d (AB), 1H, $\underline{\mathrm{H}}_{2} \mathrm{Ph}$ ); 3.52 (br s, 4H, NBD); 3.42 (br s, 2H, NBD); 1.19 (s, 2H, CH 2 NBD). ${ }^{13} \mathrm{C}\left\{{ }^{1} \mathrm{H}\right\}$ NMR (125 MHz, $\mathrm{CDCl}_{3}$ ): $\delta 143.4$ (quat $\mathrm{Ph}$ ); $136.0\left(\mathrm{~d}, \mathrm{Ph}, \mathrm{J}_{\mathrm{CP}}=13.3 \mathrm{~Hz}\right) ; 135.5(\mathrm{~d}$, quat $\mathrm{Ph}, \mathrm{J}_{\mathrm{CP}}=43.8 \mathrm{~Hz}$ ); $134.6\left(\mathrm{~d}\right.$, quat $\left.\mathrm{Ph}, \mathrm{J}_{\mathrm{CP}}=44.8 \mathrm{~Hz}\right) ; 131.4$ $75\left(\mathrm{~d}, \mathrm{Ph}, \mathrm{J}_{\mathrm{CP}}=9.4 \mathrm{~Hz}\right) ; 130.5\left(\mathrm{~d}, \mathrm{Ph}, \mathrm{J}_{\mathrm{CP}}=2.0 \mathrm{~Hz}\right) ; 129.5(\mathrm{Ph})$; $128.71(\mathrm{Ph}) ; 128.66\left(\mathrm{~d}, \mathrm{Ph}, \mathrm{J}_{\mathrm{CP}}=1.8 \mathrm{~Hz}\right) ; 127.9\left(\mathrm{~d}, \mathrm{Ph}, \mathrm{J}_{\mathrm{CP}}=10.4\right.$ $\mathrm{Hz}) ; 127.7(\mathrm{Ph}) ; 127.4\left(\mathrm{~d}, \mathrm{Ph}, \mathrm{J}_{\mathrm{CP}}=9.1 \mathrm{~Hz}\right) ; 88.6$ (d, quat $\mathrm{Cp}, \mathrm{J}_{\mathrm{CP}}$ $=19.7 \mathrm{~Hz}) ; 72.6(\mathrm{~s}$, subst $\mathrm{Cp}) ; 72.5\left(\mathrm{~d}, \mathrm{Ph}, \mathrm{J}_{\mathrm{CP}}=7.6 \mathrm{~Hz}\right) ; 70.7(\mathrm{~s}$, $\mathrm{Cp}$ ); 70.4 (d, quat $\mathrm{Cp}, \mathrm{J}_{\mathrm{CP}}=38.9 \mathrm{~Hz}$ ); 69.1 (d, subst $\mathrm{Cp}, \mathrm{J}_{\mathrm{CP}}=4.7$ ${ }_{80} \mathrm{~Hz}$ ); 60.4 (s, $\mathrm{CH}_{2} \mathrm{NBD}$ ); 53.8 (br s, CH NBD); 49.9 (br s, CH NBD); 48.1 (s, CH NBD); $40.6\left(\underline{\mathrm{CH}}_{2} \mathrm{Ph}\right) ; 31.3\left(\underline{\mathrm{CH}}_{2} \mathrm{Fc}\right) .{ }^{31} \mathrm{P}\left\{{ }^{1} \mathrm{H}\right\}$ NMR $\left(202 \mathrm{MHz}, \mathrm{CDCl}_{3}\right): \delta 20.3\left(\mathrm{~d}, \mathrm{~J}_{\mathrm{P}-\mathrm{Rh}}=147 \mathrm{~Hz}\right) . \mathrm{MS}(\mathrm{ESI})$ $m / e$ : 701,067 (M-Cl- $100 \%)$.

RhCl(NBD)(P,SEt) $\left(1^{\text {Et }}\right.$,yield: 99\%). ${ }^{1} \mathrm{H}$ NMR (500 MHz, $\left.{ }_{85} \mathrm{CDCl}_{3}\right): \delta 8.44(\mathrm{~m}, 2 \mathrm{H}, \mathrm{Ph}) ; 7.59(\mathrm{~m}, 3 \mathrm{H}, \mathrm{Ph}) ; 7.27$ (m, 3H, Ph); 6.87 (m, 2H, Ph); 4.52 (s, 1H, subst. Cp); 4.37 (br d (AB), 1H, $\mathrm{C}_{2} \mathrm{Fc}, \mathrm{J}_{\mathrm{HH}}=12 \mathrm{~Hz}$ ); 4.29 (s, 1H, subst. Cp); 4.14 (s, 1H, subst. Cp); 4.06 (br d (AB), $1 \mathrm{H}, \mathrm{CH}_{2} \mathrm{Fc}, \mathrm{J}_{\mathrm{HH}}=12 \mathrm{~Hz}$ ); 3.83 (s, 5H, Cp); 3.60 (br m, 4H, CH NBD); 3.42 (br s, 2H, CH NBD); 2.81 (m, $\left.{ }_{90} 1 \mathrm{H}, \mathrm{C}_{2} \mathrm{CH}_{3}\right) ; 2.57\left(\mathrm{~m}, 1 \mathrm{H}, \underline{\mathrm{C}}_{2} \mathrm{CH}_{3}\right) ; 1.38\left(\mathrm{t}, 1 \mathrm{H}, \mathrm{CH}_{3}, \mathrm{~J}_{\mathrm{HH}}=6.7\right.$ $\mathrm{Hz}) ; 1.22$ (s, 2H, $\left.\mathrm{CH}_{2} \mathrm{NBD}\right) .{ }^{13} \mathrm{C}\left\{{ }^{1} \mathrm{H}\right\} \mathrm{NMR}\left(125 \mathrm{MHz}, \mathrm{CDCl}_{3}\right)$ : $\delta 135.9\left(\mathrm{~d}, \mathrm{Ph}, \mathrm{J}_{\mathrm{CP}}=13.2 \mathrm{~Hz}\right) ; 135.5\left(\mathrm{~d}\right.$, quat $\left.\mathrm{Ph}, \mathrm{J}_{\mathrm{CP}}=44.3 \mathrm{~Hz}\right)$; $134.2\left(\mathrm{~d}\right.$, quat $\left.\mathrm{Ph}, \mathrm{J}_{\mathrm{CP}}=44.8 \mathrm{~Hz}\right) ; 131.4\left(\mathrm{~d}, \mathrm{Ph}, \mathrm{J}_{\mathrm{CP}}=9.5 \mathrm{~Hz}\right)$; $130.5\left(\mathrm{~d}, \mathrm{Ph}, \mathrm{J}_{\mathrm{CP}}=2.2 \mathrm{~Hz}\right) ; 128.7\left(\mathrm{~d}, \mathrm{Ph}, \mathrm{J}_{\mathrm{CP}}=1.8 \mathrm{~Hz}\right) ; 127.9$ (d, $\left.{ }_{95} \mathrm{Ph}, \mathrm{J}_{\mathrm{CP}}=10.4 \mathrm{~Hz}\right) ; 127.5\left(\mathrm{~d}, \mathrm{Ph}, \mathrm{J}_{\mathrm{CP}}=9.2 \mathrm{~Hz}\right) ; 88.8$ (d, quat $\mathrm{Cp}$, $\mathrm{J}_{\mathrm{CP}}=19.4 \mathrm{~Hz}$ ); 72.7 (s, subst $\mathrm{Cp}$ ); 72.5 (d, subst $\mathrm{Cp}, \mathrm{J}_{\mathrm{CP}}=7.7$ $\mathrm{Hz}$ ); 70.8 (s, Cp); 70.2 (d, quat Cp, $\mathrm{J}_{\mathrm{CP}}=38 \mathrm{~Hz}$ ); 69.0 (d, subst $\mathrm{Cp}, \mathrm{J}_{\mathrm{CP}}=4.8 \mathrm{~Hz}$ ); 60.7 (s, $\mathrm{CH}_{2} \mathrm{NBD}$ ); 55.3 (br s, CH NBD); 51.2 (br s, CH NBD); 48.2 (s, CH NBD); $30.8\left(\underline{\mathrm{CH}}_{2} \mathrm{Fc}\right) ; 29.7$ ((d, J $\mathrm{J}_{\mathrm{CRh}}$ $\left.{ }_{100}=3.4 \mathrm{~Hz}, \quad \underline{\mathrm{CH}}_{2} \mathrm{CH}_{3}\right) ; 13.4\left(\mathrm{CH}_{3}\right) .{ }^{31} \mathrm{P}\left\{{ }^{1} \mathrm{H}\right\} \mathrm{NMR}(202 \mathrm{MHz}$, $\left.\mathrm{CDCl}_{3}\right): \delta 21.3(\mathrm{~d}, \mathrm{JP}-\mathrm{Rh}=148 \mathrm{~Hz})$. MS (ESI) m/e: 639,049 (M$\mathrm{Cl}^{-}, 100 \%$ ).

Synthesis of $[\mathrm{Rh}(\mathrm{NBD})(\mathrm{P}, \mathrm{StBu})] \mathrm{BF}_{4}, \mathbf{2}^{{ }^{t \mathrm{Bu}}}$.In a Schlenk tube, under nitrogen, complex $\mathrm{RhCl}(\mathrm{NBD})(\mathrm{P}, \mathrm{S} t \mathrm{Bu})(0.148 \mathrm{mmol})$ was 105 dissolved in dichloromethane $(5 \mathrm{~mL})$ and $5 \mathrm{ml}$ of a water solution $\mathrm{ofNaBF}_{4}$ (20 mg, $0.178 \mathrm{mmol}$ ) was added. The organic phase was separated and dried over anhydrous sodium sulfate. After solvent evaporation, $[\mathrm{Rh}(\mathrm{NBD})(\mathrm{P}, \mathrm{S} t \mathrm{Bu})] \mathrm{BF}_{4}$ was obtained as a yellow solid. ${ }^{1} \mathrm{H}$ NMR (500 MHz, $\left.\mathrm{CDCl}_{3}\right): \delta 7.69(\mathrm{~m}, 2 \mathrm{H}, \mathrm{Ph}) ; 7.55-7.50$ $110(\mathrm{~m}, 6 \mathrm{H}, \mathrm{Ph}) ; 7.40$ (m, 2H, Ph); $5.85-5.65$ (br m, 2H, Ph, CH nbd); 4.77 (s, 1H, subst. Cp); 4.64 (s, 5H, Cp); 4.49 (m, 1H, subst. Cp); 4.35 - 4.30 (br s, 3H, CH nbd); 4.12 (m, 1H, subst. $\mathrm{Cp}) ; 3.96\left(\mathrm{~d}(\mathrm{AB}), 1 \mathrm{H}, \mathrm{CH}_{2} \mathrm{Fc}, \mathrm{J}_{\mathrm{HH}}=12.2 \mathrm{~Hz}\right) ; 3.90-3.70$ (br s, $1 \mathrm{H}, \mathrm{CH}$ nbd); 2.81 (d (AB), $1 \mathrm{H}, \mathrm{CH}_{2} \mathrm{Fc}, \mathrm{J}_{\mathrm{HH}}=12.2 \mathrm{~Hz}$ ); 1.69 (br $\left.{ }_{115} \mathrm{~s}, 2 \mathrm{H}, \mathrm{CH}_{2} \mathrm{nbd}\right) ; 1.37(\mathrm{~s}, 9 \mathrm{H}, t \mathrm{Bu}) .{ }^{13} \mathrm{C}\left\{{ }^{1} \mathrm{H}\right\} \mathrm{NMR}(125 \mathrm{MHz}$, $\left.\mathrm{CDCl}_{3}\right): \delta 134.0\left(\mathrm{~d}, \mathrm{Ph}, \mathrm{J}_{\mathrm{CP}}=13.2 \mathrm{~Hz}\right) ; 132.1\left(\mathrm{~d}, \mathrm{Ph}, \mathrm{J}_{\mathrm{CP}}=2.2 \mathrm{~Hz}\right)$; 
$131.7\left(\mathrm{~d}, \mathrm{Ph}, \mathrm{J}_{\mathrm{CP}}=9.9 \mathrm{~Hz}\right) ; 131.3\left(\mathrm{~d}, \mathrm{Ph}, \mathrm{J}_{\mathrm{CP}}=2.4 \mathrm{~Hz}\right) ; 129.6(\mathrm{dd}$, quat $\left.\mathrm{Ph}, \mathrm{J}_{\mathrm{CP}}=45.8 \mathrm{~Hz}\right) ; 129.5\left(\mathrm{~d}, \mathrm{Ph}, \mathrm{J}_{\mathrm{CP}}=10.6 \mathrm{~Hz}\right) ; 129.0(\mathrm{~d}$, $\left.\mathrm{Ph}, \mathrm{J}_{\mathrm{CP}}=10.8 \mathrm{~Hz}\right) ; 126.0\left(\mathrm{~d}\right.$, quat $\mathrm{Ph}, \mathrm{J}_{\mathrm{CP}}=52.3 \mathrm{~Hz}$ ); 85.9 (d, quat $\mathrm{Cp}, \mathrm{J}_{\mathrm{CP}}=18.5 \mathrm{~Hz}$ ); 83.8 (br s, CH nbd); 75.8 (d, subst Cp, $\mathrm{J}_{\mathrm{CP}}=$ $56.8 \mathrm{~Hz}$ ); 72.2 (s, subst Cp); 72.0 (s, CH nbd); 71.0 (s, Cp); 70.0 (br s, $\mathrm{CH}$ nbd); 68.9 (d, subst Cp, $\mathrm{J}_{\mathrm{CP}}=5.7 \mathrm{~Hz}$ ); 67.8 (d, $\mathrm{CH}_{2}$ nbd, $\left.\mathrm{J}_{\mathrm{C}-\mathrm{Rh}}=4.4 \mathrm{~Hz}\right) ; 64.7\left(\mathrm{~d}\right.$, quat. $\left.\mathrm{Cp}, \mathrm{J}_{\mathrm{CP}}=55.2 \mathrm{~Hz}\right) ; 54.8(\mathrm{~s}, \mathrm{~S}-$ $\left.\underline{\mathrm{C}}\left(\mathrm{CH}_{3}\right)\right) ; 31.0\left(\mathrm{~s}, \mathrm{~S}-\mathrm{C}\left(\underline{\mathrm{CH}}_{3}\right)\right) ; 29.1\left(\mathrm{~d}, \underline{\mathrm{CH}}_{2} \mathrm{Fc}, \mathrm{J}_{\mathrm{CP}}=5.3 \mathrm{~Hz} .{ }^{31} \mathrm{P}\right.$ NMR $\left(500202 \mathrm{MHz}, \mathrm{CDCl}_{3}\right): \delta 26.1\left(\mathrm{~d}, \mathrm{~J}_{\mathrm{P}-\mathrm{Rh}}=158 \mathrm{~Hz}\right) . \mathrm{MS}$ 10 (ESI) $m / e$ : 667,081 (M-BF $\left.{ }_{4}^{-}, 100 \%\right)$.

General procedure for the synthesis of $\mathrm{RhCl}(\mathrm{COD})(\mathrm{P}, \mathrm{SR})$, 3. In a Schlenk tube, under nitrogen, ligand ( $\mathrm{P}, \mathrm{St}$ Bu) $(0.797$ mmol) was dissolved in dichloromethane $(5 \mathrm{~mL})$ and $[\mathrm{RhCl}(\mathrm{COD})]_{2}(196 \mathrm{mg}, 0.397 \mathrm{mmol})$ was added. The solution 15 was stirred for $4 \mathrm{~h}$ at room temperature and $15 \mathrm{~mL}$ of pentane was then added to form a yellow precipitate. The precipitate was filtered under nitrogen and washed with pentane, to give $[\mathrm{RhCl}(\mathrm{COD})(\mathrm{P}, \mathrm{SR})$. Only in the case of $\mathrm{R}=t \mathrm{Bu}$, the product could be isolated in a pure state. Yield: $64 \% . \mathbf{R}=\boldsymbol{t} \mathbf{B u} .{ }^{1} \mathrm{H}$ NMR $20\left(500 \mathrm{MHz}, \mathrm{CDCl}_{3}\right): \delta 8.06(\mathrm{~m}, 2 \mathrm{H}, \mathrm{Ph}) ; 7.55-7,25(\mathrm{~m}, 8 \mathrm{H}, \mathrm{Ph})$; 4.64 (br s, $1 \mathrm{H}$, subst. Cp); 4.36 (br s, $1 \mathrm{H}$, subst. Cp); 4,35-4,20 (m, 2H, 1 subst. $\mathrm{Cp}+1 \quad \mathrm{C}_{2} \mathrm{Fc}$ ); 4.09 (s, 5H, Cp); 3.91 (br d $\left.(\mathrm{AB}), 1 \mathrm{H}, \mathrm{C}_{2} \mathrm{Fc}, \mathrm{J}_{\mathrm{HH}}=12.9 \mathrm{~Hz}\right) ; 2.48\left(\mathrm{~m}, 2 \mathrm{H}, \mathrm{CH}_{2} \operatorname{cod}\right) ; 2.32$ (m, 2H, $\mathrm{CH}_{2}$ cod); 1.99 (m, 2H, $\left.\mathrm{CH}_{2} \operatorname{cod}\right) ; 1.87$ (m, 2H, $\mathrm{CH}_{2}$ $\left.{ }_{25} \mathrm{cod}\right) ; 1.41(\mathrm{~s}, 9 \mathrm{H}, \mathrm{tBu}) .{ }^{13} \mathrm{C} \mathrm{HNMR}\left(125 \mathrm{MHz}, \mathrm{CDCl}_{3}\right): \delta 135.1$ $\left(\mathrm{d}, \mathrm{Ph}, \mathrm{J}_{\mathrm{CP}}=11.9 \mathrm{~Hz}\right) ; 134.4\left(\mathrm{~d}\right.$, quat $\left.\mathrm{Ph}, \mathrm{J}_{\mathrm{CP}}=43.6 \mathrm{~Hz}\right) ; 133.9(\mathrm{~d}$, $\left.\mathrm{Ph}, \mathrm{J}_{\mathrm{CP}}=10.5 \mathrm{~Hz}\right) ; 133.2\left(\mathrm{~d}\right.$, quat $\left.\mathrm{Ph}, \mathrm{J}_{\mathrm{CP}}=43.4 \mathrm{~Hz}\right) ; 130.1(\mathrm{~d}$, $\left.\mathrm{Ph}, \mathrm{J}_{\mathrm{CP}}=1.7 \mathrm{~Hz}\right) ; 129.5(\mathrm{Ph}) ; 127.7\left(\mathrm{~d}, \mathrm{Ph}, \mathrm{J}_{\mathrm{CP}}=10.0 \mathrm{~Hz}\right) ; 127.5$ $\left(\mathrm{d}, \mathrm{Ph}, \mathrm{J}_{\mathrm{CP}}=9.6 \mathrm{~Hz}\right) ; 90.6\left(\mathrm{~d}\right.$, quat $\left.\mathrm{Cp}, \mathrm{J}_{\mathrm{CP}}=14.1 \mathrm{~Hz}\right) ; 74.6(\mathrm{~d}$, 30 subst $\left.\mathrm{Cp}, \mathrm{J}_{\mathrm{CP}}=7.6 \mathrm{~Hz}\right) ; 74.3\left(\mathrm{~d}\right.$, quat $\left.\mathrm{Cp}, \mathrm{J}_{\mathrm{CP}}=42.2 \mathrm{~Hz}\right) ; 71.3(\mathrm{~d}$, subst. Cp, $\left.\mathrm{J}_{\mathrm{CP}}=6.9 \mathrm{~Hz}\right) ; 71.1(\mathrm{~s}, \mathrm{Cp}) ; 69.8\left(\mathrm{~d}\right.$, substCp, $\mathrm{J}_{\mathrm{CP}}=7.2$ $\mathrm{Hz}) ; 43.0$ (s, S-C( $\left.\underline{\mathrm{CH}}_{3}\right)$ ); 31.9 (v. br s, $\mathrm{CH}_{2} \operatorname{cod}$ ); 31.1 (s, S$\mathrm{C}\left(\underline{\mathrm{CH}}_{3}\right)$ ); 30.3 (v. br s, $\mathrm{CH}_{2}$ cod), 28.6 (br s, $\underline{\mathrm{CH}}_{2} \mathrm{Fc}$ ). ${ }^{31} \mathrm{P} \mathrm{NMR}$ $\left(202 \mathrm{MHz}, \mathrm{CDCl}_{3}\right): \delta 21.0\left(\mathrm{~d}, \mathrm{~J}_{\mathrm{P}-\mathrm{Rh}}=148 \mathrm{~Hz}\right) . \mathrm{MS}(\mathrm{ESI}) \mathrm{m} / \mathrm{e}$ : ${ }_{35} 683,112\left(\mathrm{M}-\mathrm{Cl}^{-}, 100 \%\right)$.

Essential spectroscopic parameters for the other compounds: $\mathbf{R}$ $=\mathbf{P h}: \delta 28.0\left(\mathrm{~d}, \mathrm{~J}_{\mathrm{P}-\mathrm{Rh}}=166 \mathrm{~Hz}\right) . \mathbf{R}=\mathbf{B z}: \delta 31.1\left(\mathrm{~d}, \mathrm{~J}_{\mathrm{P}-\mathrm{Rh}}=170\right.$ $\mathrm{Hz}) . \mathbf{R}=\mathbf{E t}: \delta 22.8\left(\mathrm{~d}, \mathrm{~J}_{\mathrm{P}-\mathrm{Rh}}=144 \mathrm{~Hz}\right)$.

General procedure for the synthesis of $40[\mathbf{R h}(\mathbf{C O D})(\mathbf{P}, \mathbf{S R})] \mathbf{B F}_{4}, 4$. In a Schlenk tube, under nitrogen, ligand (P,SR) (0.305 mmol) was dissolved in dichloromethane (5 $\mathrm{mL})$ and $\left[\mathrm{Rh}(\mathrm{COD})_{2}\right] \mathrm{BF}_{4}(124 \mathrm{mg}, 0.305 \mathrm{mmol})$ was added. The solution was stirred for $30 \mathrm{~min}$ at room temperature and $15 \mathrm{~mL}$ of pentane was then added to yield a yellow precipitate. The 45 precipitate was filtered under argon and washed with pentane to give $[\mathrm{Rh}(\mathrm{COD})(\mathrm{P}, \mathrm{SR})] \mathrm{BF}_{4}$.

$[\mathbf{R h}(\mathbf{C O D})(\mathbf{P}, \mathbf{S} t \mathbf{B u})] \mathbf{B F}_{4} \quad\left(\mathbf{4}^{t \mathrm{Bu}}\right.$, yield $\left.99 \%\right) .{ }^{1} \mathrm{H}$ NMR (500 $\left.\mathrm{MHz}, \mathrm{CDCl}_{3}\right): \delta 7.60-7.35(\mathrm{~m}, 10 \mathrm{H}, \mathrm{Ph}) ; 5.78($ br s, $2 \mathrm{H}, \mathrm{CH}$ COD); 4.78 (br s, 1H, subst. Cp); 4.64 (s, 5H, Cp); 4.48 (br s, 1H, 50 subst. Cp); 4.12 (br s, $1 \mathrm{H}$, subst. Cp); $4.00-3.95$ (m, 2H, $1 \mathrm{H} \mathrm{CH}$ $\operatorname{cod}+1 \mathrm{H} \mathrm{C} \underline{\mathrm{H}}_{2} \mathrm{Fc}$ ); 3.85 (br s, $\left.1 \mathrm{H}, \mathrm{CH} \mathrm{COD}\right) ; 2.87$ (m, 1H, $\mathrm{CH}_{2}$ $\mathrm{COD}) ; 2.71\left(\mathrm{~m}, 1 \mathrm{H}, \mathrm{CH}_{2} \mathrm{COD}\right) ; 2.69\left(\mathrm{~d}(\mathrm{AB}), 1 \mathrm{H}, \mathrm{C}_{2} \mathrm{Fc}, \mathrm{J}_{\mathrm{HH}}=\right.$ $12.2 \mathrm{~Hz}$ ); 2.59 (m, 1H, $\left.\mathrm{CH}_{2} \mathrm{COD}\right) ; 2.49$ (m, 1H, $\mathrm{CH}_{2} \mathrm{COD}$ ); 2.39 (m, 1H, $\left.\mathrm{CH}_{2} \mathrm{COD}\right) ; 2.30$ (m, 1H, $\left.\mathrm{CH}_{2} \mathrm{COD}\right) ; 2.07$ (m, 1H, $\mathrm{CH}_{2}$ $\left.{ }_{55} \mathrm{COD}\right) ; 1.96\left(\mathrm{~m}, 1 \mathrm{H}, \mathrm{CH}_{2} \mathrm{COD}\right) ; 1.37$ (s, 9H, tBu). ${ }^{13} \mathrm{C}\left\{{ }^{1} \mathrm{H}\right\} \mathrm{NMR}$ $\left(125 \mathrm{MHz}, \mathrm{CDCl}_{3}\right): \delta 133.9\left(\mathrm{~d}, \mathrm{Ph}, \mathrm{J}_{\mathrm{CP}}=12.7 \mathrm{~Hz}\right) ; 132.3(\mathrm{~d}, \mathrm{Ph}$, $\left.\mathrm{J}_{\mathrm{CP}}=2.0 \mathrm{~Hz}\right) ; 132.1\left(\mathrm{~d}, \mathrm{Ph}, \mathrm{J}_{\mathrm{CP}}=9.0 \mathrm{~Hz}\right) ; 131.6\left(\mathrm{~d}, \mathrm{Ph}, \mathrm{J}_{\mathrm{CP}}=2.2\right.$ $\mathrm{Hz}) ; 130.0\left(\mathrm{~d}\right.$, ipso $\left.\mathrm{Ph}, \mathrm{J}_{\mathrm{CP}}=44.4 \mathrm{~Hz}\right) ; 129.5\left(\mathrm{~d}, \mathrm{Ph}, \mathrm{J}_{\mathrm{CP}}=10.4\right.$
$\mathrm{Hz}) ; 128.8\left(\mathrm{~d}, \mathrm{Ph}, \mathrm{J}_{\mathrm{CP}}=10.0 \mathrm{~Hz}\right) ; 125.9\left(\mathrm{~d}\right.$, ipso $\mathrm{Ph}, \mathrm{J}_{\mathrm{CP}}=50.6$ $\left.{ }_{60} \mathrm{~Hz}\right) ; 104.4\left(\mathrm{dd}, \mathrm{CH}\right.$ COD, $\left.\mathrm{J}_{\mathrm{CP}}=9.7 \mathrm{~Hz}, \mathrm{~J}_{\mathrm{CRh}}=7.1 \mathrm{~Hz}\right) ; 102.1(\mathrm{dd}$, $\mathrm{CH}$ COD, $\left.\mathrm{J}_{\mathrm{CP}}=9.4 \mathrm{~Hz}, \mathrm{~J}_{\mathrm{CRh}}=6.1 \mathrm{~Hz}\right) ; 85.5\left(\mathrm{~d}\right.$, ipso Cp, $\mathrm{J}_{\mathrm{CP}}=$ $17.7 \mathrm{~Hz}) ; 82.1\left(\mathrm{~d}, \mathrm{CH}\right.$ COD, J $\left.\mathrm{J}_{\mathrm{CRh}}=11.3 \mathrm{~Hz}\right) ; 81.3(\mathrm{~d}, \mathrm{CH}$ COD, $\left.\mathrm{J}_{\mathrm{CRh}}=12.6 \mathrm{~Hz}\right) ; 76.1\left(\mathrm{~d}\right.$, subst $\left.\mathrm{Cp}, \mathrm{J}_{\mathrm{CP}}=6.7 \mathrm{~Hz}\right) ; 73.1$ (s, subst $\mathrm{Cp}$ ); 71.0 (s, Cp); 69.2 (s, subst Cp, $\mathrm{J}_{\mathrm{CP}}=5.7 \mathrm{~Hz}$ ); 63.2 (d, ipso $\left.{ }_{65} \mathrm{Cp}, \mathrm{J}_{\mathrm{CP}}=56.1 \mathrm{~Hz}\right) ; 55.7$ (s, S- $\underline{\mathrm{C}}\left(\mathrm{CH}_{3}\right)$ ); 35.7 (s, $\left.\mathrm{CH}_{2} \mathrm{COD}\right) ; 31.5$ $\left(\mathrm{CH}_{3}\right) ; 31.0$ (s, $\left.\mathrm{CH}_{2} \mathrm{COD}\right) ; 29.2$ (s, $\underline{\mathrm{CH}}_{2} \mathrm{Fc}$ ); 28.7 (s, $\mathrm{CH}_{2} \mathrm{COD}$ ); 27.0 (s, $\left.\mathrm{CH}_{2} \mathrm{COD}\right) .{ }^{31} \mathrm{P}\left\{{ }^{1} \mathrm{H}\right\} \mathrm{NMR}\left(202 \mathrm{MHz}, \mathrm{CDCl}_{3}\right): \delta 23.8(\mathrm{~d}$,

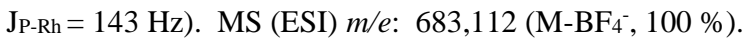

$[\mathbf{R h}(\mathbf{C O D})(\mathbf{P}, \mathbf{S P h})] \mathbf{B F}_{4} \quad\left(\mathbf{4}^{\mathbf{P h}}\right.$, yield: $\left.80 \%\right) .{ }^{1} \mathrm{H}$ NMR $(500$ $70 \mathrm{MHz}_{\mathrm{CDCl}}$ ): $\delta$ 7.80-7.35 (m, 15H, Ph); 5.17 (m, 1H, CH COD); 4.72 (br s, 1H, subst. Cp); 4.70 (s, 5H, Cp), 4.49 (br s, 1H, subst. $\mathrm{Cp}) ; 4.29$ (m, 1H, CH COD); 4.17 (d (AB), 1H, $\underline{\mathrm{H}}_{2} \mathrm{Fc}, \mathrm{J}_{\mathrm{HH}}=$ $12.3 \mathrm{~Hz}$ ); 4.12 (m, 1H, CH COD); 4.09 (br s, 1H, subst. Cp); $4.01(\mathrm{~m}, 1 \mathrm{H}, \mathrm{CH} \mathrm{COD}) ; 3.04\left(\mathrm{~d}(\mathrm{AB}), 1 \mathrm{H}, \mathrm{C}_{2} \mathrm{Fc}, \mathrm{J}_{\mathrm{HH}}=12.3\right.$ $75 \mathrm{~Hz}$ ); 2.80 (m, 1H, CH $2 \mathrm{COD}) ; 2.68$ (m, 1H, $\left.\mathrm{CH}_{2} \mathrm{COD}\right), 2.46$ (m, $\left.2 \mathrm{H}, \mathrm{CH}_{2} \mathrm{COD}\right) ; 2.36$ (m, 1H, $\left.\mathrm{CH}_{2} \mathrm{COD}\right) ; 2.14$ (m, 1H, $\mathrm{CH}_{2}$ COD) $2.04\left(\mathrm{~m}, 1 \mathrm{H}, \mathrm{CH}_{2} \mathrm{COD}\right) 1.96\left(\mathrm{~m}, 1 \mathrm{H}, \mathrm{CH}_{2} \mathrm{COD}\right) .{ }^{13} \mathrm{C}\left\{{ }^{1} \mathrm{H}\right\}$ NMR (125 MHz, $\left.\mathrm{CDCl}_{3}\right): \delta 134.1\left(\mathrm{~d}, \mathrm{Ph}, \mathrm{J}_{\mathrm{CP}}=12.7 \mathrm{~Hz}\right) ; 132.7$ $(\mathrm{Ph}) ; 131.9\left(\mathrm{~d}, \mathrm{Ph}, \mathrm{J}_{\mathrm{CP}}=9.4 \mathrm{~Hz}\right) ; 131.7(\mathrm{Ph}) ; 131.3(\mathrm{Ph}) ; 130.4$ $80(\mathrm{Ph}) ; 130.4$ (d, ipso $\left.\mathrm{Ph}, \mathrm{J}_{\mathrm{CP}}=45.1 \mathrm{~Hz}\right) ; 129.9\left(\mathrm{~d}, \mathrm{Ph}, \mathrm{J}_{\mathrm{CP}}=10.1\right.$ $\mathrm{Hz}$ ); 125.9 (d, ipso $\mathrm{Ph}, \mathrm{J}_{\mathrm{CP}}=49.5 \mathrm{~Hz}$ ); 107.0 (br s, CH COD); $103.3\left(\mathrm{dd}, \mathrm{CH} \mathrm{COD}, \mathrm{J}_{\mathrm{CP}}=9.0 \mathrm{~Hz}, \mathrm{~J}_{\mathrm{CRh}}=6.0 \mathrm{~Hz}\right) ; 86.2(\mathrm{~d}, \mathrm{CH}$ $\left.\mathrm{COD}, \mathrm{J}_{\mathrm{CRh}}=11.8 \mathrm{~Hz}\right) ; 84.8\left(\mathrm{~d}\right.$, ipso $\left.\mathrm{Cp}, \mathrm{J}_{\mathrm{CRh}}=16.9 \mathrm{~Hz}\right) ; 84.1(\mathrm{~d}$, CH COD, $\left.\mathrm{J}_{\mathrm{CRh}}=10.8 \mathrm{~Hz}\right) ; 75.9\left(\mathrm{~d}\right.$, subst $\left.\mathrm{Cp}, \mathrm{J}_{\mathrm{CP}}=6.2 \mathrm{~Hz}\right) ; 72.9$ ${ }_{85}$ (br s, subst Cp); 71.3 (br s, Cp); 69.1 (d, subst Cp, J $\mathrm{JP}_{\mathrm{CP}}=5.8 \mathrm{~Hz}$ ); 64.6 (d, ipso Cp, J $\mathrm{JP}=55.9 \mathrm{~Hz}) ; 37.9\left(\mathrm{~d}, \mathrm{CH}_{2} \mathrm{Fc}, \mathrm{J}_{\mathrm{CP}}=4.6 \mathrm{~Hz}\right)$; 34.4 (s, $\mathrm{CH}_{2} \mathrm{COD}$ ); 30.3 (s, $\mathrm{CH}_{2} \mathrm{COD}$ ); 30.0 (s, $\mathrm{CH}_{2} \mathrm{COD}$ ); 27.1 (s, $\left.\mathrm{CH}_{2} \mathrm{COD}\right) .{ }^{31} \mathrm{P}\left\{{ }^{1} \mathrm{H}\right\}$ NMR $\left(202 \mathrm{MHz}, \mathrm{CDCl}_{3}\right): \delta 22.1$ (d, JP-Rh $=144 \mathrm{~Hz})$. MS (ESI) m/e: 703,081 (M-BF4- $100 \%)$.

$90 \quad[\mathbf{R h}(\mathbf{C O D})(\mathbf{P}, \mathbf{S B z})] \mathbf{B F} 4 \mathbf{4}\left(4^{\mathrm{Bz}}\right.$, yield $\left.42 \%\right) .{ }^{1} \mathrm{H}$ NMR $(500 \mathrm{MHz}$, $\left.\mathrm{CDCl}_{3}\right): \delta 7.70(\mathrm{~m}, 2 \mathrm{H}, \mathrm{Ph}) ; 7.60-7.50(\mathrm{~m}, 8 \mathrm{H}, \mathrm{Ph}) ; 7.34(\mathrm{~m}, 5 \mathrm{H}$, $\mathrm{Ph}) ; 5.57$ (m, 2H, CH COD); 4.54 (br s, 6H, Cp + 1H subst. Cp); 4.42 (br s, $1 \mathrm{H}$, subst. Cp); 4.19 (d (AB), $1 \mathrm{H}, \mathrm{C}_{2} \mathrm{Ph}, \mathrm{J}_{\mathrm{HH}}=13.8$ $\mathrm{Hz}$ ); 4.13 (br s, $1 \mathrm{H}$, subst. $\mathrm{Cp}$ ); 4.03 (d (AB), $1 \mathrm{H}, \mathrm{C}_{2} \mathrm{Ph}, \mathrm{J}_{\mathrm{HH}}=$ $\left.{ }_{95} 13.8 \mathrm{~Hz}\right) ; 3.97$ (m, 2H, CH COD); 3.50 (d (AB), $1 \mathrm{H}, \mathrm{CH}_{2} \mathrm{Fc}, \mathrm{J}_{\mathrm{HH}}$ $=12.5 \mathrm{~Hz}) ; 2.92(\mathrm{~m}, 1 \mathrm{H}, \mathrm{COD}), 2.76(\mathrm{~m}, 1 \mathrm{H}, \mathrm{CH}$ COD$) ; 2.67(\mathrm{~d}$ $\left.(\mathrm{AB}), 1 \mathrm{H}, \mathrm{CH}_{2} \mathrm{Fc}, \mathrm{J}_{\mathrm{HH}}=12.5 \mathrm{~Hz}\right) ; 2.60\left(\mathrm{~m}, 2 \mathrm{H}, \mathrm{CH}_{2} \mathrm{COD}\right) ; 2.48$ (m, 2H, $\left.\mathrm{CH}_{2} \mathrm{COD}\right) 2.36\left(\mathrm{~m}, 2 \mathrm{H}, \mathrm{CH}_{2} \mathrm{COD}\right) 2.13\left(\mathrm{~m}, 2 \mathrm{H}, \mathrm{CH}_{2}\right.$ COD). ${ }^{13} \mathrm{C}\left\{{ }^{1} \mathrm{H}\right\}$ NMR $\left(125 \mathrm{MHz}, \mathrm{CDCl}_{3}\right): \delta 134.2\left(\mathrm{~d}, \mathrm{Ph}, \mathrm{J}_{\mathrm{CP}}=\right.$ $10012.9 \mathrm{~Hz}$ ); 133.7 (quat $\mathrm{Ph}) ; 132.3\left(\mathrm{~d}, \mathrm{Ph}, \mathrm{J}_{\mathrm{CP}}=9.8 \mathrm{~Hz}\right) ; 132.1$ (d, $\left.\mathrm{Ph}, \mathrm{J}_{\mathrm{CP}}=2.3 \mathrm{~Hz}\right) ; 131.4\left(\mathrm{~d}, \mathrm{Ph}, \mathrm{J}_{\mathrm{CP}}=2.3 \mathrm{~Hz}\right) ; 130.2$ (d, quat $\mathrm{Ph}$, $\left.\mathrm{J}_{\mathrm{CP}}=46.2 \mathrm{~Hz}\right) ; 129.8\left(\mathrm{~d}, \mathrm{Ph}, \mathrm{J}_{\mathrm{CP}}=10.5 \mathrm{~Hz}\right) ; 129.5(\mathrm{Ph}) ; 129.1$ $(\mathrm{Ph}) ; 128.8\left(\mathrm{~d}, \mathrm{Ph}, \mathrm{J}_{\mathrm{CP}}=10.11 \mathrm{~Hz}\right) ; 127.9\left(\mathrm{~d}, \mathrm{Ph}, \mathrm{J}_{\mathrm{CP}}=10.4 \mathrm{~Hz}\right)$; $128.4(\mathrm{Ph}) ; 127.2$ (d, quat $\mathrm{Ph}, \mathrm{J}_{\mathrm{CP}}=49.6 \mathrm{~Hz}$ ); 105.8 (dd, CH COD, $\left.105 \mathrm{~J}_{\mathrm{CP}}=9.6 \mathrm{~Hz}, \mathrm{~J}_{\mathrm{CRh}}=7.1 \mathrm{~Hz}\right) ; 102.7\left(\mathrm{dd}, \mathrm{CH} \mathrm{COD}, \mathrm{J}_{\mathrm{CP}}=9.9 \mathrm{~Hz}\right.$, $\left.\mathrm{J}_{\mathrm{CRh}}=6.5 \mathrm{~Hz}\right) ; 85.3\left(\mathrm{~d}, \mathrm{CH} \mathrm{COD}, \mathrm{J}_{\mathrm{CRh}}=10.9 \mathrm{~Hz}\right) ; 85.1(\mathrm{~d}, \mathrm{CH}$ $\mathrm{COD}, \mathrm{J}_{\mathrm{CRh}}=11.6 \mathrm{~Hz}$ ); 75.4 (br s, subst Cp); 72.6 (br s, subst Cp); 71.4 (br s, Cp); 69.2 (br s, subst Cp); $42.6\left(\mathrm{CH}_{2} \mathrm{Ph}\right) ; 34.2$ (d, $\mathrm{CH}_{2}$ $\left.\mathrm{COD}, \mathrm{J}_{\mathrm{CP}}=3.5 \mathrm{~Hz}\right) ; 31.5\left(\mathrm{~d}, \underline{\mathrm{CH}}_{2} \mathrm{Fc}, \mathrm{J}_{\mathrm{CP}}=4.6 \mathrm{~Hz}\right) ; 31.0(\mathrm{~d}$, $\left.{ }_{110} \mathrm{CH}_{2} \mathrm{COD}, \mathrm{J}_{\mathrm{CP}}=3.5 \mathrm{~Hz}\right) ; 29.7\left(\mathrm{~d}, \mathrm{CH}_{2} \mathrm{COD}, \mathrm{J}_{\mathrm{CP}}=3.5 \mathrm{~Hz}\right) ; 27.6$ (d, $\left.\mathrm{CH}_{2} \mathrm{COD}, \mathrm{J}_{\mathrm{CP}}=3.5 \mathrm{~Hz}\right) .{ }^{31} \mathrm{P}\left\{{ }^{1} \mathrm{H}\right\} \mathrm{NMR}\left(202 \mathrm{MHz}, \mathrm{CDCl}_{3}\right): \delta$ $21.8\left(\mathrm{~d}, \mathrm{~J}_{\mathrm{P}-\mathrm{Rh}}=144 \mathrm{~Hz}\right)$.

[Rh(COD)(P,SEt)]BF 4 (4 ${ }^{\text {Et }}$, yield: $\left.76 \%\right) .{ }^{1} \mathrm{H}$ NMR (500 MHz, $\left.\mathrm{CDCl}_{3}\right): \delta 7.77-7.62(\mathrm{~m}, 2 \mathrm{H}, \mathrm{Ph}) ; 7.61-7.35(\mathrm{~m}, 8 \mathrm{H}, \mathrm{Ph}) ; 5.43$ 115 (m, 1H, CH COD); 5.33 (m, 1H, CH COD); 4.74 (br s, 1H, subst. $\mathrm{Cp}) ; 4.65$ (s, 5H, Cp); 4.48 (m, 1H, subst. Cp); 4.12 (br s, $1 \mathrm{H}$, 
subst. Cp); $4.06-3.82\left(\mathrm{~m}, 3 \mathrm{H}, 2 \mathrm{H} \mathrm{CH} \operatorname{cod}+1 \mathrm{H} \underline{\mathrm{H}}_{2} \mathrm{Fc}\right) ; 2.99$ (m, $\left.1 \mathrm{H}, \mathrm{CH}_{2} \mathrm{CH}_{3}\right) ; 2.92-2.80$ (m, 1H, $\left.\mathrm{CH}_{2} \mathrm{COD}\right) ; 2.80-2.25$ (m, 5H, $\left.\mathrm{CH}_{2} \mathrm{COD}\right) ; 2.70$ (d (AB), $1 \mathrm{H}, \mathrm{C}_{2} \mathrm{Fc}, \mathrm{J}_{\mathrm{HH}}=12.6 \mathrm{~Hz}$ ); $2.20-2.00$ (m, 2H, CH $2 \mathrm{COD}) ; 1.32\left(\mathrm{t}, 3 \mathrm{H}, \mathrm{CH}_{3}, \mathrm{~J}_{\mathrm{HH}}=7.2 \mathrm{~Hz}\right)$. ${ }_{5}^{13} \mathrm{C}\left\{{ }^{1} \mathrm{H}\right\} \mathrm{NMR}\left(125 \mathrm{MHz}, \mathrm{CDCl}_{3}\right): \delta 134.2\left(\mathrm{~d}, \mathrm{Ph}, \mathrm{J}_{\mathrm{CP}}=13.0 \mathrm{~Hz}\right)$; $132.3\left(\mathrm{~d}, \mathrm{Ph}, \mathrm{J}_{\mathrm{CP}}=2.2 \mathrm{~Hz}\right) ; 132.0\left(\mathrm{~d}, \mathrm{Ph}, \mathrm{J}_{\mathrm{CP}}=9.5 \mathrm{~Hz}\right) ; 131.5(\mathrm{~d}$, $\left.\mathrm{Ph}, \mathrm{J}_{\mathrm{CP}}=2.4 \mathrm{~Hz}\right) ; 130.2\left(\mathrm{~d}\right.$, quat $\left.\mathrm{Ph}, \mathrm{J}_{\mathrm{CP}}=45.4 \mathrm{~Hz}\right) ; 129.6(\mathrm{~d}, \mathrm{Ph}$, $\left.\mathrm{J}_{\mathrm{CP}}=10.5 \mathrm{~Hz}\right) ; 128.8\left(\mathrm{~d}, \mathrm{Ph}, \mathrm{J}_{\mathrm{CP}}=10.0 \mathrm{~Hz}\right) ; 129.1(\mathrm{Ph}) ; 126.5(\mathrm{~d}$, quat $\left.\mathrm{Ph}, \mathrm{J}_{\mathrm{CP}}=49.3 \mathrm{~Hz}\right) ; 104.7\left(\mathrm{dd}, \mathrm{CH} \mathrm{COD}, \mathrm{J}_{\mathrm{CP}}=9.6 \mathrm{~Hz}, \mathrm{~J}_{\mathrm{CRh}}=\right.$ $107.3 \mathrm{~Hz}$ ); $103.3\left(\mathrm{dd}, \mathrm{CH}\right.$ COD, $\left.\mathrm{J}_{\mathrm{CP}}=10.0 \mathrm{~Hz}, \mathrm{~J}_{\mathrm{CRh}}=6.2 \mathrm{~Hz}\right) ; 85.8$ (d, quat $\left.\mathrm{Cp}, \mathrm{J}_{\mathrm{CP}}=16.1 \mathrm{~Hz}\right) ; 84.9\left(\mathrm{~d}, \mathrm{CH} \mathrm{COD}, \mathrm{J}_{\mathrm{CRh}}=11.7 \mathrm{~Hz}\right)$; $83.9\left(\mathrm{~d}, \mathrm{CH}\right.$ COD, $\left.\mathrm{J}_{\mathrm{CRh}}=10.9 \mathrm{~Hz}\right) ; 75.7\left(\mathrm{~d}\right.$, subst $\mathrm{Cp}, \mathrm{J}_{\mathrm{CP}}=5.9$ $\mathrm{Hz}$ ); 72.6 (s, subst Cp); 71.2 (s, Cp); 69.0 (s, subst Cp, $\mathrm{J}_{\mathrm{CP}}=5.4$ $\mathrm{Hz}) ; 34.6\left(\mathrm{~d}, \mathrm{CH}_{2} \mathrm{COD}, \mathrm{J}_{\mathrm{CP}}=3.5 \mathrm{~Hz}\right) ; 32.8\left(\underline{\mathrm{CH}}_{2} \mathrm{CH}_{3}\right) ; 30.9(\mathrm{~s}$, ${ }_{15} \mathrm{CH}_{2} \mathrm{COD}$ ); 30.3 (d, $\underline{\mathrm{CH}}_{2} \mathrm{Fc}, \mathrm{J}_{\mathrm{CP}}=5.1 \mathrm{~Hz}$ ); 29.5 (s, $\mathrm{CH}_{2} \mathrm{COD}$ );

Table 1.

Crystallographic data (excluding structure factors) have been deposited with the Cambridge Crystallographic Data Centre as supplementary publication no. CCDC 872062 for $\mathbf{2}^{\mathbf{t B u}}$, no. CCDC
$15.0\left(\mathrm{CH}_{3}\right) .{ }^{31} \mathrm{P}\left\{{ }^{1} \mathrm{H}\right\} \mathrm{NMR}\left(500202 \mathrm{MHz}, \mathrm{CDCl}_{3}\right): \delta 22.9\left(\mathrm{~d}, \mathrm{~J}_{\mathrm{P}-\mathrm{Rh}}\right.$ $=144 \mathrm{~Hz})$. MS (ESI) m/e: 655,081 (M-BF $\left.{ }_{4}^{-}, 100 \%\right)$.

X-ray crystallography. A single crystal of each compound was mounted under inert perfluoropolyether on the tip of a 20 cryoloop and cooled in the cryostream of either an OxfordDiffraction XCALIBUR SAPPHIRE-I CCD diffractometer or an Agilent Technologies GEMINI EOS CCD diffractometer. Data were collected using the monochromatic MoK $\alpha$ radiation $(\lambda=$ 0.71073). The structures were solved by direct methods 25 (SIR97) $^{37}$ and refined by least-squares procedures on $F^{2}$ using SHELXL-97. ${ }^{38}$ All $\mathrm{H}$ atoms attached to carbon were introduced in idealised positions and treated as riding on their parent atoms in the calculations. The drawing of the molecules was realised with the help of ORTEP $3{ }^{39}, 40$ Crystal data and refinement 30 parameters are shown in

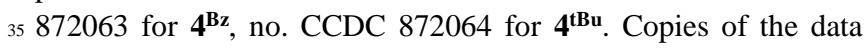
can be obtained free of charge on application to the Director, CCDC, 12 Union Road, Cambridge CB2 1EZ, UK (fax: (+44) 1223-336-033; e-mail: deposit@ccdc.cam.ac.uk).

Table 1. Crystal data and structure refinement parameters.

\begin{tabular}{|c|c|c|c|}
\hline & $2^{t \mathrm{Bu}}$ & $4^{\mathrm{Ph}}$ & $4^{\mathrm{Bz}}$ \\
\hline Empiricalformula & $\mathrm{C}_{34} \mathrm{H}_{37} \mathrm{FePRhS}, \mathrm{BF}_{4}$ & $\mathrm{C}_{37} \mathrm{H}_{37} \mathrm{FePRhS}, \mathrm{BF}_{4}, \mathrm{CH}_{2} \mathrm{Cl}_{2}$ & $\mathrm{C}_{38} \mathrm{H}_{39} \mathrm{FePRhS}, \mathrm{BF}_{4}, \mathrm{CH}_{2} \mathrm{Cl}_{2}$ \\
\hline Formula weight & 754.24 & 875.19 & 889.22 \\
\hline Wavelength, $\AA$ & 0.71073 & 0.71073 & 0.71073 \\
\hline Crystal system & Orthorhombic & Orthorhombic & Triclinic \\
\hline Space group & $\mathrm{P} 2_{1} 2_{1} 2_{1}$ & $\mathrm{Pca}_{1}$ & $P-1$ \\
\hline$c, \AA$ & 17.435 & $14.209(5)$ & $13.5655(8)$ \\
\hline$\alpha,^{\circ}$ & 90 & 90 & $108.445(6)$ \\
\hline$\beta,^{\circ}$ & 90 & 90 & $101.880(5)$ \\
\hline$\gamma,^{\circ}$ & 90 & 90 & $98.703(5)$ \\
\hline Volume, $\AA^{3}$ & 3157.3 & $3610(2)$ & $1813.9(2)$ \\
\hline$F(000)$ & 1536 & 1776 & 904 \\
\hline Crystal size, $\mathrm{mm}^{3}$ & $0.54 \times 0.47 \times 0.18$ & $0.56 \times 0.22 \times 0.07$ & $0.43 \times 0.22 \times 0.16$ \\
\hline 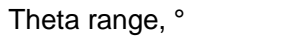 & 3.02 to 27.10 & 2.98 to 26.36 & 3.12 to 26.37 \\
\hline Reflections collected & 12237 & 19753 & 14216 \\
\hline Indpt reflections $\left(\mathrm{R}_{\text {int }}\right)$ & $6118(0.0344)$ & $7239(0.0342)$ & $7393(0.0506)$ \\
\hline Completeness, \% & 98.5 & 99.8 & 99.8 \\
\hline Absorption correction & Multi-scan & Multi-scan & Multi-scan \\
\hline Max. and min. transm. & 1.0 and 0.619 & 1.0 and 0.7726 & 1.0 and 0.77764 \\
\hline Refinement method & $\mathrm{F}^{2}$ & $\mathrm{~F}^{2}$ & \\
\hline Data /restr./param. & $6118 / 24 / 388$ & 7250 / 1 / 442 & 7408 / 24 / 478 \\
\hline Goodness-of-fit on $F^{2}$ & 1.094 & 1.037 & 1.039 \\
\hline
\end{tabular}

40

Computational details. Calculations were performed with the Gaussian09 package $^{41}$ using the B3LYP hybrid functional ${ }^{42,} 43$ under the DFT approach, since it performs better in frequency
45 calculations. All carbon and hydrogen atoms were described with the $6-31 \mathrm{G}(\mathrm{d}, \mathrm{p})$ basis set, whereas the $6-31++\mathrm{G}(\mathrm{d}, \mathrm{p})$ basis set was applied to the $\mathrm{P}, \mathrm{S}, \mathrm{B}, \mathrm{F}$ and $\mathrm{Cl}$ atoms. Effective core potentials (ECP) and its associated SDD basis set ${ }^{44-47}$ supplemented with $\mathrm{f}$ - 
polarization functions $(\operatorname{SDD}(\mathrm{f}))^{48}$ were applied for the $\mathrm{Rh}$, Ir and Fe atoms. Geometry optimizations were performed for the neutral NBD and COD complexes $\mathbf{1}$ and $\mathbf{3}$ with $\mathrm{R}=\mathrm{Ph}$ and $t \mathrm{Bu}$ without any ligand simplification. Calculations were also carried out on 5 the isolated cation of $\mathbf{2}^{\mathbf{P h}}$ and on the iridium analogue $\operatorname{IrCl}(\mathrm{COD})(\mathrm{P}, \mathrm{St} \mathrm{Bu})$. No scaling factors were applied to the calculated low-frequency vibrations. Since the inclusion of anharmonicity effects in the case of $3^{\text {tBu }}$ did not lead to any significant change in the $\mathrm{Rh}-\mathrm{Cl}$ frequency values (see Supporting 10 Information, Table S1), this method was not applied to the other calculations.

\section{General procedure of asymmetric hydrogenation}

Solution of $6.4 * 10^{-3} \mathrm{mmol}$ of catalyst, $3.2 * 10^{-2} \mathrm{mmol}$ of $\mathrm{CH}_{3} \mathrm{ONa}$ (5 equivs.) and $3.2 \mathrm{mmol}$ of acetophenone (500 equivs.) in $2 \mathrm{ml}$ 15 of isopropanol was transferred into a 5-ml glass vial which was then placed under argon into a stainless steel autoclave. The reaction vessel was pressurized to the 30 bar of $\mathrm{H}_{2}$ pressure and stirred with magnetic bar for 1 hour at room temperature. The reaction was stopped by release of pressure. The crude materials

20 were obtained by purification reaction mixture by chromatography on silica using dichloromethane as an eluent. The product was finally analyzed by chiral GC.

\section{Results and Discussion.}

\section{(a) Syntheses}

${ }_{25}$ The addition of the (P,SR) ligand (1 equiv per $\mathrm{Rh}$ atom) to a dichloromethane solution of $[\mathrm{RhCl}(\mathrm{NBD})]_{2}$ or $[\mathrm{RhCl}(\mathrm{COD})]_{2}$ yielded the corresponding adducts $\mathrm{RhCl}(\mathrm{NBD})(\mathrm{P}, \mathrm{SR}), \mathbf{1}^{\mathbf{R}}$, and $\mathrm{RhCl}(\mathrm{COD})(\mathrm{P}, \mathrm{SR}), \mathbf{3}^{\mathbf{R}}$, see Scheme 2. For the NBD reagent, the complete series with $\mathrm{R}=t \mathrm{Bu}, \mathrm{Ph}, \mathrm{Bz}$ and Et was obtained as pure 30 derivatives as shown by the NMR and HRMS analyses. Concerning the COD series, although all compounds could be obtained in solution as shown by the spectroscopic analyses, only the $t \mathrm{Bu}$ derivative $3^{t \mathrm{Bu}}$ could be isolated as a pure product. Starting from $\mathbf{1}^{\mathbf{t} \mathbf{B u}}$, addition of $\mathrm{NaBF}_{4}$ in dichloromethane led to 35 the precipitation of $\mathrm{NaCl}$ and formation of $[\mathrm{Rh}(\mathrm{NBD})(\mathrm{P}, \mathrm{S} t \mathrm{Bu})] \mathrm{BF}_{4}, \mathbf{2}^{t \mathbf{B u}}$, in sufficient purity. The complete series of the $\mathrm{BF}_{4}$ salts containing the COD ligand, $[\mathrm{Rh}(\mathrm{COD})(\mathrm{P}, \mathrm{SR})] \mathrm{BF}_{4}, \mathbf{4}^{\mathbf{R}}$, on the other hand, was more conveniently obtained by addition of $(\mathrm{P}, \mathrm{SR})$ to a solution of 40 compound $\left[\mathrm{Rh}(\mathrm{COD})_{2}\right] \mathrm{BF}_{4}$. All these were isolated in a pure state.

\section{(b) Characterization of the $[\mathrm{Rh}(\text { diene })(\mathrm{P}, \mathrm{SR})]^{+}$salts 2 and 4}

We begin the characterization section with the salts $\mathbf{2}$ and $\mathbf{4}$, for which the analysis is more straightforward. The NMR spectra ${ }^{45}$ show standard ${ }^{1} \mathrm{H},{ }^{13} \mathrm{C}$ and ${ }^{31} \mathrm{P}$ chemical shifts and coupling patterns for the expected square planar coordination around the $\mathrm{Rh}^{\mathrm{I}}$ center. In particular, $\mathrm{Rh}$ coupling is visible for selected ${ }^{13} \mathrm{C}$ resonances of the diene ligand and for the phosphine ${ }^{31} \mathrm{P}$ resonance (the latter is collected in Table 2 for all compounds).

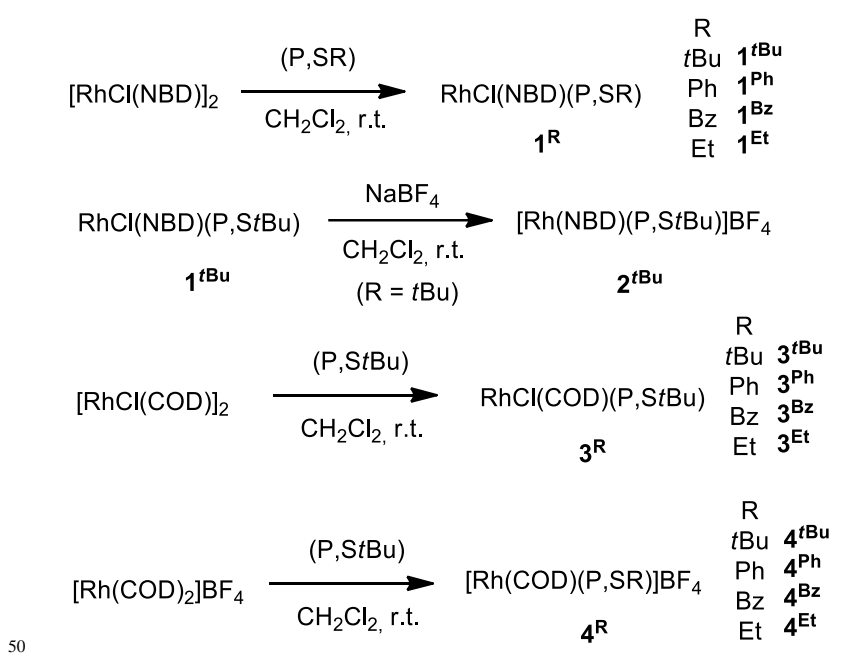

Scheme 2. Summary of the synthetic work and compounds numbering scheme.

Table 2. ${ }^{31} \mathrm{P}$ NMR data for the Rh complexes ( $\delta$ in ppm with the ${ }^{2} J_{\mathrm{PRh}}$ in Hertz in parentheses).

\begin{tabular}{lcccc}
\hline & $\mathbf{1}^{\mathbf{R}}$ & $\mathbf{2}^{\mathbf{R}}$ & $\mathbf{3}^{\mathbf{R}}$ & $\mathbf{4}^{\mathbf{R}}$ \\
\hline $\mathrm{R}=t \mathrm{Bu}$ & $23.6(159)$ & $26.1(158)$ & $21.0(148)$ & $23.8(143)$ \\
$\mathrm{R}=\mathrm{Ph}$ & $23.8(154)$ & & $28.0(166)$ & $22.1(144)$ \\
$\mathrm{R}=\mathrm{Bz}$ & $20.3(147)$ & & $31.1(170)$ & $21.8(144)$ \\
$\mathrm{R}=\mathrm{Et}$ & $21.3(148)$ & & $22.8(144)$ & $22.9(144)$ \\
\hline
\end{tabular}

55

The molecular geometry of compounds $2^{t \mathbf{B u}}, \mathbf{4}^{\mathrm{Ph}}$ and $\mathbf{4}^{\mathrm{Bz}}$ was confirmed by single crystal X-ray diffraction. Whereas compound $2^{t \mathbf{B u}}$ contains only the cation and the anion in the crystal, compounds $4^{\mathrm{Ph}}$ and $4^{\mathrm{Bz}}$ crystallize with one molecule of ${ }_{60}$ dichloromethane per ion pair. Views of the three cations are shown in Figure 1 and relevant bond distances and angles for the three compounds are compared in Table 3. The geometry is typical of $\left[\mathrm{Rh}(\text { diene })\left(\mathrm{L}^{1} \mathrm{~L}^{2}\right)\right]^{+}$complexes where $\mathrm{L}^{1} \mathrm{~L}^{2}$ is a chelating ligand, of which over 1000 are reported in the literature. ${ }_{65}$ The two midpoints of the donating ene functions and the $\mathrm{P}$ and $\mathrm{S}$ donor atoms define an approximate square planar configuration, which is quite typical of $d^{8} \mathrm{Rh}^{\mathrm{I}}$. As a matter of fact, the geometry is nearly for $4^{\mathrm{Ph}}$, with trans angles quite close to $180^{\circ}(\mathrm{P}-\mathrm{Rh}-\mathrm{X} 1$ and $\mathrm{S}-\mathrm{Rh}-\mathrm{X} 2=178.6(3)$ and $174.69(6)^{\circ}$, respectively; $\mathrm{X}=$ 70 midpoints of the $\mathrm{C}=\mathrm{C}$ bonds, see Table 3 ), whereas it is more distorted for $4^{\mathrm{Bz}}$. A close inspection of the structure of $4^{\mathrm{Bz}}$ shows that this distortion is related to a twist of the COD ligand, which is caused by a van der Waals repulsion between one of the COD $\mathrm{CH}=\mathrm{CH}$ donating groups $(\mathrm{C} 11-\mathrm{C} 12)$ and the $\mathrm{CH}_{2}$ group of the 75 benzyl substituent on sulfur (C22). A similar van der Waals repulsion, namely a $2.16 \AA$ contact between the H atoms on one of the $t \mathrm{Bu}$ methyl groups $(\mathrm{C} 23)$ and on atom $\mathrm{C} 12$ of the NBD ligand, explains the even larger distortion observed in $\mathbf{2}^{\mathbf{t B u}}(\mathrm{P}-\mathrm{Rh}$ $\mathrm{X} 1$ and $\mathrm{S}-\mathrm{Rh}-\mathrm{X} 2=163.54(4)$ and $166.20(4)^{\circ}$, respectively; see 80 Table 3). As expected, the X1-Rh-X2 angle is much smaller for the NBD ligand with a value of $69.943(11)^{\circ}$, similar to the angle observed in related compound. ${ }^{29}$ Another peculiar geometrical feature is that the sulfur substituent is placed anti relative to the $\mathrm{CpFe}$ moiety of the ferrocene group for the three complexes. ${ }_{85}$ Upon coordination, the sulfur atom becomes chiral and therefore two different diastereoisomers could be obtained in principle, with the sulfur substituent either syn or anti to the CpFe moiety. 
Like for all previously reported complexes containing ligands of this family, without exception, ${ }^{31,}{ }^{49-51}$ a single compound is obtained in solution and the sulphur substituent is placed on the side opposite (anti) to the $\mathrm{FeCp}$ group with respect to the S-C-C${ }_{5} \mathrm{C}$-P chelate. Consequently, the observed diastereomer has the configuration $\left(S_{\mathrm{fc}}, S_{\mathrm{S}}\right)$ or $\left(R_{\mathrm{fc}}, R_{\mathrm{S}}\right)$ for $2^{t \mathrm{Bu}}$ and for $4^{\mathrm{Ph}}$ and $\left(R_{\mathrm{fc}}, S_{\mathrm{S}}\right)$ or $\left(S_{\mathrm{fc}}, R_{\mathrm{S}}\right)$ for $\mathbf{4}^{\mathrm{Bz}}$. The two $\mathrm{Cp}$ rings are roughly eclipsed with the largest twist angle, $\tau, 7.5(4)^{\circ}$ for $4^{\mathrm{Bz}}$.
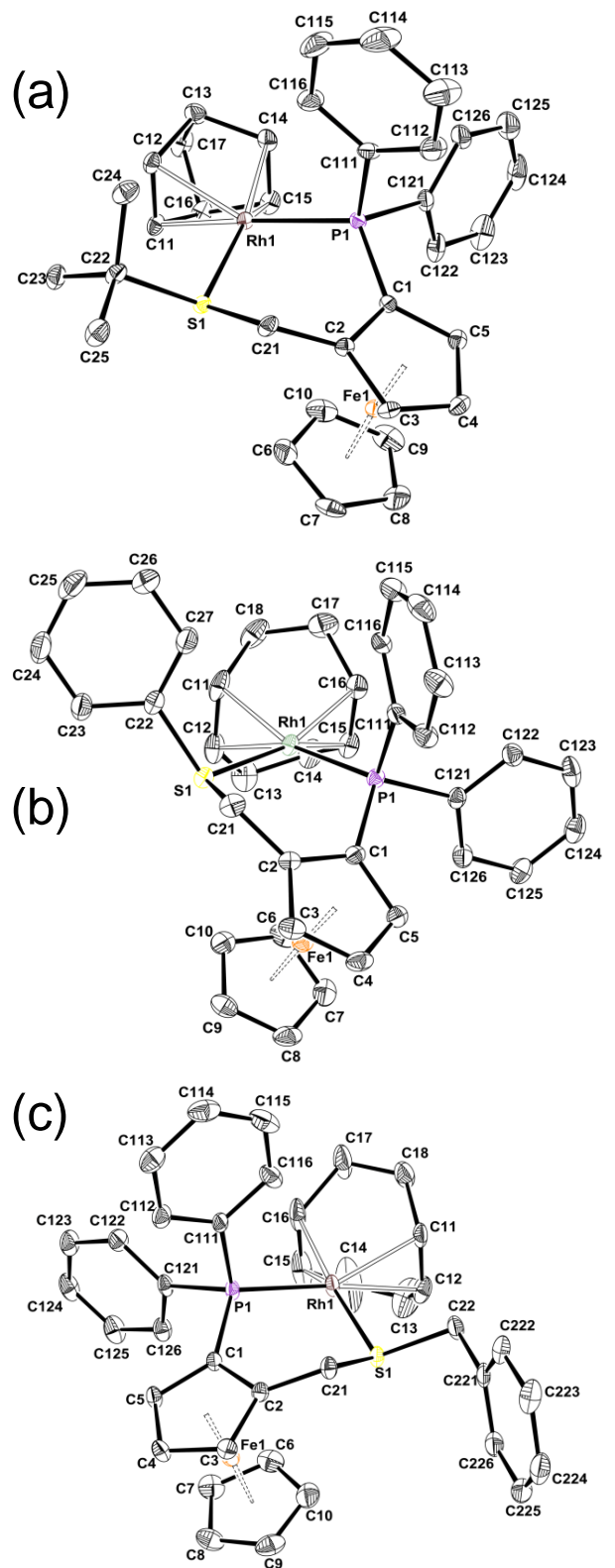

10 Figure 1. Molecular views of the cationic complexes in compounds $\mathbf{2}^{\mathbf{B u}}$

(a), $\mathbf{4}^{\mathrm{Ph}}$ (b) and $\mathbf{4}^{\mathrm{Bz}}$ (c) with the atom-labelling scheme. Displacement ellipsoids are drawn at the $30 \%$ probability level. The $\mathrm{BF}_{4}{ }^{-}$counterion and $\mathrm{H}$ atoms have been omitted for clarity.

A few other $[\mathrm{Rh}(\text { diene })(\mathrm{P}, \mathrm{S})]^{\mathrm{n}+}$ complexes of $d^{8} \mathrm{Rh}^{\mathrm{I}}$ have been 15 previously described, some being cationic with a phosphinethioether ${ }^{28,29,52-55}$ or phosphine-phosphine sulfide ${ }^{56,57}$ ligand, others being neutral with a phosphine-thiolato ligand, ${ }^{56,58,59}$ but the Rh-S distance does not appear to be too sensitive to this modification. The Rh-P and Rh-S distances found for $\mathbf{4}^{\mathrm{Ph}}$ and $\mathbf{4}^{\mathrm{Bz}}$ 20 (see Table 3 ) compare quite well with the average in the above mentioned examples (Rh-P: 2.29(3) $\AA$ and Rh-S: 2.36(3) $\AA$ ). Whereas the Rh-P distances are quite similar for the three compounds, the $\mathrm{Rh}-\mathrm{S}$ distance is significantly shorter in $\mathbf{4}^{\mathrm{Bz}}$, suggesting a better binding ability of the benzyl derivative, and 25 longer in $\mathbf{2}^{\mathbf{t} \mathbf{B u}}$, probably reflecting the greater steric bulk of the sulphur substituent which is also responsible for the greater geometry distortion (vide supra). There are no reasons to doubt that the solution structures of the other $4^{\mathbf{R}}$ compounds are identical to the solid state structure found for $4^{\mathrm{Ph}}$ and $\mathbf{4}^{\mathrm{Bz}}$.

30 DFT/B3LYP geometry optimizations were carried out for the cations of $2^{\mathbf{R}}$ and $\mathbf{4}^{\mathbf{R}}$ with $\mathrm{R}=t \mathrm{Bu}$ and $\mathrm{Ph}$. The results are also reported in Table 3. For compounds $2^{t \mathbf{B u}}$ and $\mathbf{4}^{\mathbf{P h}}$, the optimized geometry reproduced quite well the experimentally observed one, except for a slight overestimation of the Rh-P, Rh-S and Rh-C 35 distances (by $0.08 \AA$ on average for Rh-S and Rh-P, $0.04 \AA$ for Rh-C). Whereas the calculated Rh-P distance is essentially the same in all four compounds, the Rh-S distance increases from $\mathrm{Ph}$ to the more bulky $t \mathrm{Bu}$ substituent, in agreement with the experimental evidence. Views of all optimized geometries are 40 provided in the Supporting Information, Figure S1.

\section{(c) Characterization of the chloride complexes 1 and 3}

Concerning the neutral chlorido complexes, the solution and solid state structures cannot be assigned as easily because of the presence of five potentially coordinating ligands. We were 45 unfortunately unable to obtain single crystals for any of these compounds, preventing us to determine the solid state structure. Interestingly, the structure adopted by the related iridium complexes $\operatorname{IrCl}(\mathrm{COD})\left\{\mathrm{CpFe}\left[1,2-\mathrm{C}_{5} \mathrm{H}_{3}\left(\mathrm{PPh}_{2}\right)\left(\mathrm{CH}_{2} \mathrm{SR}\right)\right]\right\}$ depends on the nature of the thioether substituent. ${ }^{31}$ For $\mathrm{R}=t \mathrm{Bu}$, the 50 molecule adopts a square planar geometry with a (COD)ClP coordination environment and a dangling thioether function (i.e. structure I in Scheme 3), whereas the compounds with $\mathrm{R}=\mathrm{Et}, \mathrm{Ph}$ and $\mathrm{Bz}$ prefer a pentacoordinated square pyramidal geometry with a loosely bonded axial $\mathrm{Cl}$ ligand (structure II). These 55 structural motifs seem to be maintained in solution, according to observed differences in the NMR properties, notably the ${ }^{31} \mathrm{P}$ resonance. ${ }^{31}$ The $\mathrm{Rh}$ complexes $\mathbf{1}^{\mathbf{R}}$ and $\mathbf{3}^{\mathbf{R}}$ could also conceivably adopt structure I or II, but a third possibility (III) with a chelating $(\mathrm{P}, \mathrm{S})$ ligand and an outer sphere chloride ion, related to ${ }_{60}$ those observed for $\mathbf{2}^{t \mathbf{B u}}, \mathbf{4}^{\mathrm{Ph}}$ and $\mathbf{4}^{\mathrm{Bz}}$ by simple replacement of the $\mathrm{BF}_{4}^{-}$ion with $\mathrm{Cl}^{-}$, can also be imagined.

Inspection of the previously published X-ray structures of related rhodium complexes suggests that III is unlikely but cannot be totally excluded, because a handful of compounds with ${ }_{65}$ the $\mathrm{RhX}($ diene $)\left(\mathrm{L}^{1} \mathrm{~L}^{2}\right)$ stoichiometry feature indeed a free halide anion. In these structures, the cationic complex is characterized by strongly binding neutral ligands such as two monodentate $\mathrm{N}$ heterocyclic carbenes (NHC), ${ }^{60}$ a bidentatebis-NHC, ${ }^{61-63}$ a $2,2^{2}-$ bipyridine, ${ }^{64}$ or a 2,2 '-dipyridylmethane. ${ }^{65}$ All the other structures 70 of this type contain, like those of $\mathbf{4}^{\mathrm{Ph}}$ and $\mathbf{4}^{\mathrm{Bz}}$, non coordinating $\mathrm{X}^{-}$ anion (mostly $\mathrm{PF}_{6}^{-}, \mathrm{BF}_{4}^{-}, \mathrm{CF}_{3} \mathrm{SO}_{3}^{-}$and $\mathrm{ClO}_{4}^{-}$). A 5-coordinate geometry of type II also appears unlikely because it is much less common for $\mathrm{Rh}^{\mathrm{I}}$ than for $\mathrm{Ir}^{\mathrm{I}}$, but again it cannot be excluded. Rare examples of 5-coordinate $\mathrm{RhX}($ diene $)\left(\mathrm{L}^{1} \mathrm{~L}^{2}\right)$, with COD or NBD 75 and including a halide ligand in the coordination geometry are limited to complexes where $\mathrm{L}^{1} \mathrm{~L}^{2}$ is bipyridine, ${ }^{64}$ 2-phenyl- 
azopyridine, ${ }^{64}$ 2-pyridylimines, ${ }^{66}$ or an NHC-oxazoline. ${ }^{67-69}$ It is also relevant to compare pairs of related $\left[\mathrm{Rh}(\text { diene })\left(\mathrm{L}^{1} \mathrm{~L}^{2}\right)\right]^{+} \mathrm{X}^{-}$ and $\mathrm{RhY}($ diene $)\left(\mathrm{L}^{1} \mathrm{~L}^{2}\right)$ structures $(\mathrm{X}=$ non coordinating anion, $\mathrm{Y}$ $=$ halide), where the weaker partner of the chelating $\mathrm{L}^{1} \mathrm{~L}^{2}$ donor 5 set dissociates to let the halide enter the coordination sphere on going from the former to the latter. Examples of this situation include bidentate ligands $\mathrm{L}^{1} \mathrm{~L}^{2}$ such as phosphine-amines, ${ }^{70}, 71$ phosphine-ethers, ${ }^{72}$ or phosphine-phosphine oxides. ${ }^{73,}{ }^{74}$ Clearly, all these examples involve a relatively strong donor (e.g. a 10 phosphine) and a weaker one ( $\mathrm{N}$ or $\mathrm{O}$ donor) giving the bidentate ligand a pronounced hemilability character. Apparently, examples of this type for phosphine-thioethers have not yet been described. Since no suitable single crystals for a diffraction analysis could be obtained for any of compounds $\mathbf{1}$ and $\mathbf{3}$, their structure was 15 addressed by a combination of spectroscopic techniques (IR, NMR) and DFT calculations.

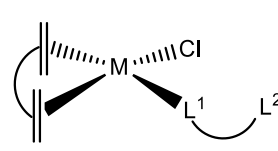

I

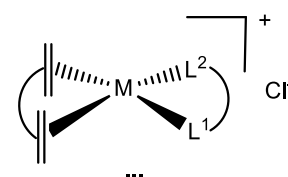

III
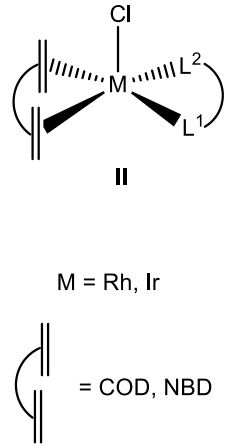

Scheme 3. Possible molecular geometries for $\mathrm{MCl}($ diene $)\left(\mathrm{L}^{1} \mathrm{~L}^{2}\right)$ complexes $(\mathrm{M}=\mathrm{Rh}, \mathrm{Ir})$.

20 As shown in Table 2, the ${ }^{31} \mathrm{P}$ chemical shift and Rh coupling for compounds $\mathbf{1}$ and $\mathbf{3}$ do not greatly differ from those of the $\mathrm{BF}_{4}^{-}$salts 2 and 4 . This is an expected occurrence if the chloride complexes adopted structure III. It should be remarked that the ${ }^{31} \mathrm{P}$ chemical shift in the $[\operatorname{IrCl}(\mathrm{COD})(\mathrm{P}, \mathrm{SR})]$ and
$25[\operatorname{Ir}(\mathrm{COD})(\mathrm{P}, \mathrm{SR})]^{+}$compounds was found diagnostic for discriminating structures of type $\mathbf{I}(\delta-4.2$ for $\mathrm{R}=\mathrm{Ph}$ and -3.1 for $\mathrm{R}=\mathrm{Et})$, II $(\delta 15.7$ for $\mathrm{R}=t \mathrm{Bu})$ and III $(\delta 11.0$ for $\mathrm{R}=\mathrm{Ph}$ and 14.5 for $\mathrm{R}=t \mathrm{Bu}) .{ }^{31}$ In particular, the ${ }^{31} \mathrm{P}$ resonance is rather similar for the Ir complexes of type II and III and different 30 relative to those with structure I. Therefore, the similarity between the resonances of the chloride complexes $\mathbf{1}$ and $\mathbf{3}$ with those of the $\mathrm{BF}_{4}$ salts $\mathbf{2}$ and $\mathbf{4}$ that are known to adopt structure III would appear to point to a structure of type II or III for the chloride complexes. This argument, however, can in no way be 35 considered conclusive because the metal nature may have a different effect on the chemical shift of the different structural types.

Another useful comparison comes from the detailed analysis of the ${ }^{1} \mathrm{H}$ spectra. Most protons (i.e. diene, $\mathrm{PPh}_{2}$ ) are not expected to 40 greatly respond to the structural type. The $\mathrm{FcCH}_{2} \mathrm{~S}$ protons, on the other hand, may be sensitive to the thioether coordination, namely discriminate between I and II/III. The two protons of this group, whether the $\mathrm{S}$ atom is coordinated or not, are diastereotopic and therefore always give rise to an $\mathrm{AB}$ pattern.

${ }_{45}$ This feature was also observed for the free ligands and for the sulfur-protected version of the free ligand, $(\mathrm{S}=\mathrm{P}, \mathrm{SR}),{ }^{23}$ the ${ }^{1} \mathrm{H}$ and ${ }^{13} \mathrm{C}$ resonances of which are collected in Table 4 together with those of the isolated compounds $\mathbf{1 - 4}$. It can be noted that the two doublets of the $\mathrm{AB}$ pattern in the ${ }^{1} \mathrm{H}$ NMR are very close to each 50 other in the $(\mathrm{P}, \mathrm{SR})$ and $(\mathrm{S}=\mathrm{P}, \mathrm{SR})$ molecules $(\Delta \delta<0.4 \mathrm{ppm})$. This difference is systematically much greater for the cationic complexes in the $\mathrm{BF}_{4}^{-}$salts where the $\mathrm{S}$ atom is coordinated to the Rh centre ( $0.83 \mathrm{ppm}$ in $\mathbf{4}^{\mathrm{Bz}}$ and $>1 \mathrm{ppm}$ for all other examples). It can be argued that the enantioselective sulfur coordination 55 makes a further magnetic discrimination of the two $\mathrm{CH}_{2}$ protons, thereby increasing their chemical shift difference. Considering now the chlorido derivatives, we note that three of them exhibit a small chemical shift difference $\left(0.13\right.$ for $\mathbf{1}^{\mathbf{B z}}, 0.31$ for $\mathbf{1}^{\mathbf{E t}}$, ca. 0.3 for $3^{t \mathbf{B u}}$ ), whereas the $\Delta \delta$ values are much greater for the other

60 Table 3. Selected experimental (from X-ray diffraction) and computed (by DFT optimizations) bond lengths $[\AA]$ and angles $\left[{ }^{\circ}\right]$ in compounds $2^{\mathrm{R}}$ and $4^{\mathrm{R}}$.

\begin{tabular}{|c|c|c|c|c|c|c|c|}
\hline & \multicolumn{2}{|c|}{$2^{t \mathrm{Bu}}$} & \multirow{2}{*}{$\begin{array}{c}2^{\mathrm{Ph}} \\
\text { DFT }\end{array}$} & \multirow{2}{*}{$\begin{array}{c}4^{t \mathrm{Bu}} \\
\mathrm{DFT}\end{array}$} & \multicolumn{2}{|c|}{$4^{\mathrm{Ph}}$} & \multirow{2}{*}{$\begin{array}{c}4^{\mathrm{Bz}} \\
\text { X-ray }\end{array}$} \\
\hline & X-ray & DFT & & & X-ray & DFT & \\
\hline \multicolumn{8}{|l|}{ Distances $^{\mathrm{a}}$} \\
\hline Rh-P & $2.2587(13)$ & 2.331 & 2.343 & 2.354 & $2.2858(15)$ & 2.354 & $2.2796(10)$ \\
\hline $\mathrm{Rh}-\mathrm{S}$ & $2.3641(13)$ & 2.452 & 2.402 & 2.508 & $2.3378(11)$ & 2.421 & $2.3194(10)$ \\
\hline Rh-X1 & $2.1231(3)$ & 2.165 & 2.145 & 2.179 & $2.119(2)$ & 2.184 & $2.1198(3)$ \\
\hline $\mathrm{Rh}-\mathrm{X} 2$ & $2.0066(4)$ & 2.032 & 2.047 & 2.062 & $2.040(5)$ & 2.069 & $2.0199(3)$ \\
\hline Rh-C11 & $2.229(5)$ & 2.263 & 2.260 & 2.302 & $2.227(4)$ & 2.310 & $2.210(4)$ \\
\hline Rh-C12 & $2.229(5)$ & 2.283 & 2.248 & 2.270 & $2.227(4)$ & 2.271 & $2.240(4)$ \\
\hline Rh-C15 & $2.118(5)$ & 2.159 & 2.169 & 2.173 & $2.169(4)$ & 2.182 & $2.127(5)$ \\
\hline Rh-C16 & $2.127(5)$ & 2.141 & 2.159 & 2.183 & $2.142(4)$ & 2.188 & $2.142(5)$ \\
\hline $\mathrm{C} 11-\mathrm{C} 12$ & $1.358(8)$ & 1.409 & 1.404 & 1.408 & $1.368(8)$ & 1.404 & $1.352(8)$ \\
\hline $\mathrm{C} 15-\mathrm{C} 16$ & $1.384(8)$ & 1.383 & 1.385 & 1.384 & $1.391(7)$ & 1.385 & $1.380(11)$ \\
\hline \multicolumn{8}{|l|}{ Angles $^{\mathrm{a}}$} \\
\hline P-Rh-S & $92.64(5)$ & 92.2 & 92.6 & 90.3 & $92.30(3)$ & 90.9 & $93.24(4)$ \\
\hline P-Rh-X1 & $163.54(4)$ & 165.6 & 168.8 & 175.8 & $178.6(3)$ & 177.2 & $168.29(3)$ \\
\hline P-Rh-X2 & $93.84(13)$ & 97.6 & 99.6 & 92.5 & $93.01(6)$ & 93.5 & $91.59(3)$ \\
\hline S-Rh-X1 & 103.01(3) & 102.0 & 98.5 & 93.1 & 88.83(12) & 91.0 & $90.95(3)$ \\
\hline $\mathrm{S}-\mathrm{Rh}-\mathrm{X} 2$ & $166.20(4)$ & 163.5 & 166.1 & 171.6 & $174.69(6)$ & 172.6 & $168.21(3)$ \\
\hline $\mathrm{X} 1-\mathrm{Rh}-\mathrm{X} 2$ & $69.943(11)$ & 69.2 & 69.6 & 84.4 & $85.87(17)$ & 84.9 & $86.423(13)$ \\
\hline
\end{tabular}

${ }^{\mathrm{a}} \mathrm{X} 1$ and $\mathrm{X} 2$ are the midpoints of the coordinating COD C=C functions, $\mathrm{C} 11-\mathrm{C} 12$ and $\mathrm{C} 14-\mathrm{C} 15$ (for the NBD complexes) or $\mathrm{C} 15-\mathrm{C} 16$ (for the COD complexes), respectively. 
Table 4. Selected ${ }^{1} \mathrm{H}\left(\Delta \delta\right.$ in parentheses) and ${ }^{13} \mathrm{C}$ NMR (in italics) data for the $\mathrm{FcCH}_{2} \mathrm{~S}$ group in complexes $1-4$ ( $\delta$ in ppm).

\begin{tabular}{|c|c|c|c|c|c|c|}
\hline & $1^{\mathrm{R}}$ & $2^{\mathrm{R}}$ & $3^{\mathrm{R}}$ & $4^{\mathrm{R}}$ & $(\mathrm{P}, \mathrm{SR})^{\mathrm{a}}$ & $(\mathrm{S}=\mathrm{P}, \mathrm{SR})^{\mathrm{a}}$ \\
\hline \multirow{2}{*}{$\mathrm{R}=t \mathrm{Bu}$} & $5.06,3.84(1.22)$ & $3.96,2.81(1.15)$ & ca. 4.2, 3.91 (ca. 0.3) & ca. 4.0, 2.69(са. 1.3) & $3.80,3.63(0.17)$ & $3.99,3.96(0.04)$ \\
\hline & 28.8 & 29.1 & 28.6 & 29.2 & 27.4 & 26.7 \\
\hline \multirow{2}{*}{$\mathrm{R}=\mathrm{Ph}$} & $5.37,4.2(1.09)$ & & & $4.17,3.04(1.15)$ & $4.17,4.09(0.08)$ & $4.42,4.35(0.07)$ \\
\hline & 34.1 & & & 37.9 & 34.2 & 33.3 \\
\hline \multirow{2}{*}{$\mathrm{R}=\mathrm{Bz}$} & $4.13,4.00(0.13)$ & & & $3.50,2.67(0.83)$ & $3.72,3.65(0.07)$ & $4.14,3.77(0.37)$ \\
\hline & 31.3 & & & 31.5 & 31.1 & 30.5 \\
\hline \multirow{2}{*}{$\mathrm{R}=\mathrm{Et}$} & $4.37,4.06(0.31)$ & & & ca. $4.0,2.70$ (ca. 1.3$)$ & $3.74,3.74(0.00)$ & $4.09,3.87(0.22)$ \\
\hline & 30.8 & & & 30.3 & 30.9 & 30.3 \\
\hline
\end{tabular}

${ }^{\mathrm{a}}$ Data from ref. ${ }^{23}$

two compounds (1.22 for $\mathbf{1}^{\text {tBu }}, 1.09$ for $\mathbf{1}^{\mathbf{P h}}$ ) and comparable to those of the cationic complexes. These results would tend to 5 suggest that the thioether function is not coordinated to the Rh center in complexes $\mathbf{1}^{\mathbf{B z}}, \mathbf{1}^{\mathrm{Et}}$ and $\mathbf{3}^{\text {tBu }}$ (e.g. structure $\left.\mathbf{I}\right)$, whereas coordination might occur for the other two derivatives. Analysis of the $\mathrm{FcCH}_{2} \mathrm{~S}{ }^{13} \mathrm{C}$ chemical shift does not bring any additional clarification, since this resonance appears to be very little 10 sensitive to the chemical environment as shown in Table 4.

Since the NMR analysis does not allow an unambiguous assignment of the chemical structure for the chlorido derivatives, additional studies were carried out by solid state IR spectroscopy, although limited to the COD- $\left(\mathrm{P}, \mathrm{S}^{t \mathrm{Bu}}\right)$ and NBD- $\left(\mathrm{P}, \mathrm{S}^{t \mathrm{Bu}}\right)$ 15 derivatives. Spectra in the lower fingerprint region (600-250 $\mathrm{cm}^{-1}$ ) were measured for $\mathbf{1}^{\mathrm{tBu}}, \quad \mathbf{2}^{\mathrm{tBu}}, \quad \mathbf{3}^{\mathrm{tBu}}, \quad \mathbf{4}^{\mathrm{tBu}}$ and $\operatorname{IrCl}(\mathrm{COD})(\mathrm{P}, \mathrm{St} \mathrm{Bu})$ in the solid state and the results are reported in Figure 2. The $\mathrm{M}-\mathrm{Cl}$ stretching region shows various bands, the most intense one having a higher frequency for the iridium 20 compound $\operatorname{IrCl}(\mathrm{COD})(\mathrm{P}, \mathrm{S} t \mathrm{Bu})\left(295 \mathrm{~cm}^{-1}\right)$ than for the rhodium compounds $\mathbf{1}^{\text {tBu }}$ and $\mathbf{3}^{\text {tBu }}$ (271 and $288 \mathrm{~cm}^{-1}$, respectively). The assignment of these bands to the $\mathrm{Ir}-\mathrm{Cl}$ and $\mathrm{Rh}-\mathrm{Cl}$ stretching vibrations, respectively, was confirmed by the calculations (vide infra). On going to the tetrafluoroborate salts $\mathbf{2}^{\mathbf{t B u}}$ and $\mathbf{4}^{\mathrm{tBu}}$, the 25 major band disappears in agreement with the removal of the $\mathrm{Cl}^{-}$ ligand from the metal coordination sphere. These observations are in favor of a coordination geometry of type I or II (Scheme 3).

Geometry optimizations for all three types of structures were 30 carried out at the same computational level as for the cationic complexes described above. The calculations were carried out for complexes $\mathbf{1}^{\mathbf{R}}$ and $3^{\mathbf{R}}$ for $\mathrm{R}=t \mathrm{Bu}$ and $\mathrm{Ph}$ in order to evaluate the effect of the $\mathrm{R}$ substituent and the diene ligand. The geometry of the iridium analogue $\mathrm{IrCl}(\mathrm{COD})(\mathrm{P}, \mathrm{S} t \mathrm{Bu})$ was also optimized and 35 was found to agree quite well with that obtained experimentally in the solid state. ${ }^{31} \mathrm{~A}$ table with the most significant metric parameters for all optimized structures is reported in the Supporting Information (Table S2). All attempts to optimize the geometry of $[\mathrm{Rh}(\text { diene })(\mathrm{P}, \mathrm{SR})]^{+}$with $\mathrm{Cl}^{-}$as a counteranion (e.g. a 40 geometry of type III in Scheme 3) led to the dissociation of the thioether arm to allow formation of the rhodium-chloride bond (i.e. an optimized geometry of type $\mathbf{I}$ in Scheme 3). The geometry of the only local energy minimum obtained for $\mathbf{3}^{\mathbf{t B u}}$ is quite similar to that of the iridium analogue, with the sulfur atom far 45 away from the $\mathrm{Rh}$ center $(5.556 \AA) .{ }^{31}$ Similar optimized geometries are also adopted by the other complexes $\left(1^{\mathbf{P h}}, \mathbf{1}^{\mathbf{t B u}}\right.$, $\left.\mathbf{3}^{\mathbf{P h}}\right)$. Replacement of the $S$ atom in the coordination sphere of $2^{\mathbf{R}}$ or $4^{\mathbf{R}}$ by the $\mathrm{Cl}$ atom in $\mathbf{1}^{\mathbf{R}}$ or $3^{\mathbf{R}}$ systematically lengthens the M-P bond by ca. $0.05 \AA$ ( $c f$. Table 3 and Table S2).

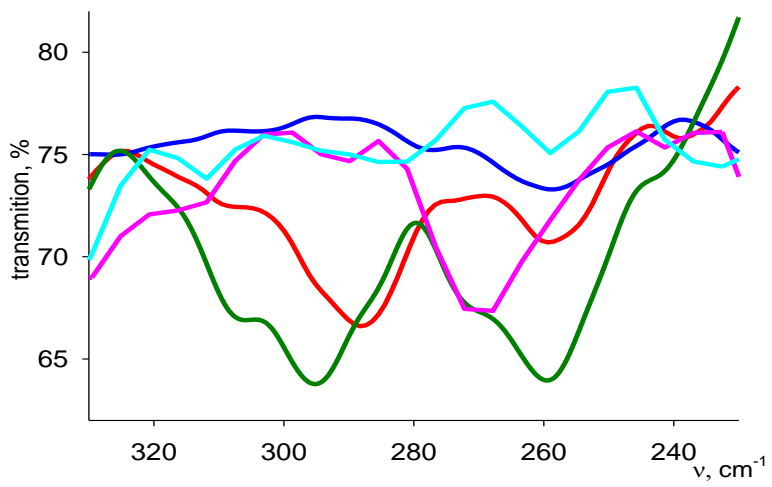

Figure 2. Solid state IR spectra (lower fingerprint region) of $\mathbf{3}^{\mathbf{t B u}}$ (red line), $\mathbf{4}^{\mathrm{tBu}}$ (blue line), $\mathbf{1}^{\mathrm{tBu}}$ (pink line), $\mathbf{2}^{\mathrm{tBu}}$ (cyan line) and $\mathrm{IrCl}(\mathrm{COD})(\mathrm{P}, \mathrm{St} \mathrm{Bu})$ (green line).

A second, higher energy minimum was also found for 55 complexes $\mathbf{1}^{\mathbf{P h}}, \mathbf{1}^{\mathbf{t B u}}$ and $\mathbf{3}^{\mathbf{P h}}$. In these structures, the sulfur donor function has rearranged to place itself along the $\mathrm{z}$ direction perpendicularly to the square plane defined by the other four ligands, loosely interacting with the metal center, defining a pseudo pentacoordinated square-pyramidal geometry like that of ${ }_{60}$ II in Scheme 3, except that the axial coordination position of the square pyramid is occupied by the sulfur atom instead of the $\mathrm{Cl}$ atom (type II'). An example is shown in Figure 3 for compound $\mathbf{1}^{t \mathbf{B u}}$ (all other optimized geometries are available in the Supporting Information section, Figure S2). The Rh-S distance is ${ }_{65}$ too long to be considered an interaction for compound $3^{\text {Ph }}(3.674$ $\AA$ ), whereas it signals a genuine 5-coordinate geometry for $\mathbf{1}^{\mathbf{P h}}$ $(2.824 \AA)$ and $\mathbf{1}^{t \mathbf{B u}}(2.702 \AA)$, when considering that the axial interaction for 5-coordinate square pyramidal $d^{8}$ complexes is lengthened by the $d_{\mathrm{z} 2}$ electron pair. The shorter Rh-S separation 70 for the $t \mathrm{Bu}$ derivative may be related to the greater donating power of the $\mathrm{S} t \mathrm{Bu}$ donor function. On the other hand, this geometry is more destabilized relative to the square planar global minimum for $\mathbf{1}^{\mathbf{t} \mathbf{B u}}$ (by $\left.8.2 \mathrm{kcal} / \mathrm{mol}\right)$ than for $\mathbf{1}^{\mathbf{P h}}(3.1 \mathrm{kcal} / \mathrm{mol})$. The greater steric bulk of the $t \mathrm{Bu}$ group may be responsible for 75 this difference. All attempts to optimize a type II geometry led to one of the above mentioned minima. Therefore, the geometry optimizations are in favor of a structure of type $\mathbf{I}$ for compounds $\mathbf{1}$ and $\mathbf{3}$, irrespective of the nature of the R substituent (at least for $\mathrm{Ph}$ and $t \mathrm{Bu})$. 


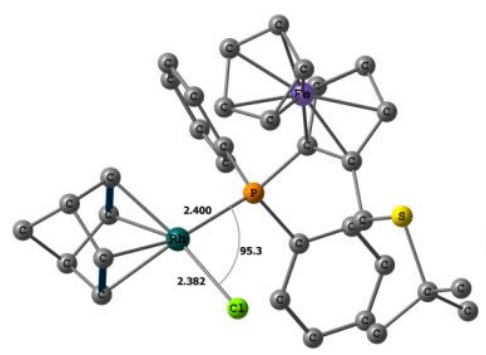

Type I (0.0)

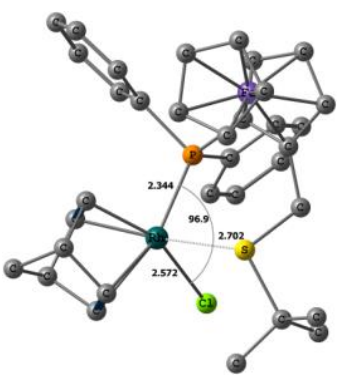

Type II' (8.2)
Figure 3. DFT(B3LYP) optimized geometries of $1^{\mathrm{Ph}}$ with relative energies in parentheses in $\mathrm{kcal} / \mathrm{mol}$. Hydrogen atoms are omitted for clarity.

5 It is now of interest to analyze the calculated IR spectrum of the observed minima and compare it with the experimental ones reported in Figure 2. The $\mathrm{M}-\mathrm{Cl}$ stretching vibration is coupled with $\mathrm{M}$-(diene) stretches in all rhodium complexes and contributes mostly to two intense bands, which exhibit a greater 10 frequency difference in the COD series. The analysis is rather simple for the COD compound $\mathbf{3}^{t \mathbf{B u}}$ and for the iridium analogue, since only a minimum of type I was optimized. The computed spectra for these two compounds, as well as for the salt $\mathbf{4}^{t \mathbf{B u}}$, are shown in Figure 4. These spectra match rather closely those 15 experimentally observed, confirming the Type I structure. The calculated maximum absorption for the strongest $\mathrm{M}-\mathrm{Cl}$ stretching band is at $279 \mathrm{~cm}^{-1}$ for the Ir complex and at $277 \mathrm{~cm}^{-1}$ for the $\mathrm{Rh}$ complex $3^{t \mathbf{B u}}$, whereas the spectrum of $4^{t \mathbf{B u}}$ does not show any strong band in this region. The adoption of a structure of type I by 20 compound $\mathbf{3}^{\boldsymbol{t} \mathrm{Bu}}$ is also in line with the suggestion of the ${ }^{1} \mathrm{H}$ NMR spectrum ( $\mathrm{AB}$ pattern of the $\mathrm{CH}_{2} \mathrm{~S} t \mathrm{Bu}$ moiety, vide supra).

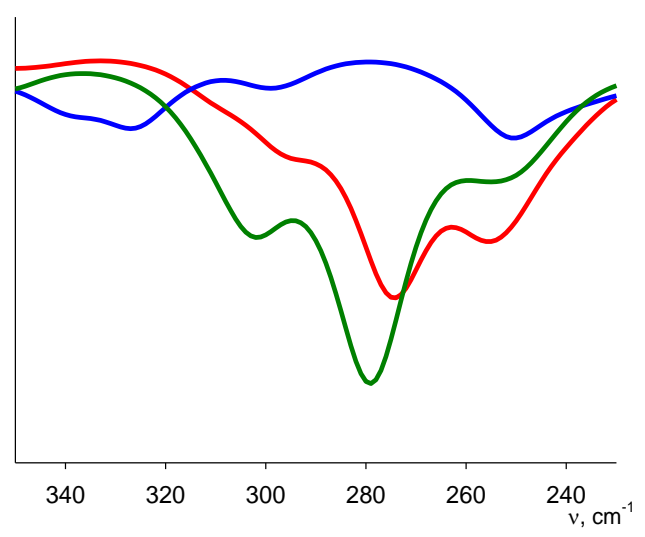

Figure 4. Calculated IR spectra (lower fingerprint region) of $\mathbf{3}^{\mathbf{t B u}}$ (red line), $4^{\text {tBu }}$ (blue line) and $\mathrm{IrCl}(\mathrm{COD})(\mathrm{P}, \mathrm{St} \mathrm{Bu})$ (green line).

25 The calculated spectra for the type I and type II' minima of compounds $1^{t \mathbf{B u}}$ and $\mathbf{1}^{\mathrm{Ph}}$ are shown in Figure 5. It can be seen that the $\mathrm{Rh}-\mathrm{Cl}$ stretching band for the type I minima is very similar to that of compound $3^{t \mathbf{B u}}$ (286 and $273 \mathrm{~cm}^{-1}$ for the strongest absorption). On the other hand, the $\mathrm{Rh}-\mathrm{Cl}$ vibration is shifted to 30 much lower frequencies in the type II' minima of $\mathbf{1}^{\mathbf{P h}}\left(247 \mathrm{~cm}^{-1}\right)$, and especially of $\mathbf{1}^{t \mathbf{B u}}\left(208 \mathrm{~cm}^{-1}\right)$. This effect can obviously be attributed to the coordination of the sulfur atom, which provides additional electron density to the metal center and labilizes the $\mathrm{Rh}-\mathrm{Cl}$ bond, an effect which is stronger for the $t \mathrm{Bu}$ derivative
35 where the Rh-S distance is shorter. In agreement with this argument, the $\mathrm{Rh}-\mathrm{Cl}$ distance is shorter for the type I minima of compounds $\mathbf{1}^{\text {tBu }}$ and $\mathbf{1}^{\mathbf{P h}}$ (2.382 and $2.390 \AA$, respectively) and longer for the type II' geometries of $\mathbf{1}^{\mathbf{P h}}(2.459 \AA)$ and particularly $\mathbf{1}^{\mathbf{t} \mathbf{B u}}(2.572 \AA)$. The difference between the calculated ${ }_{40} \mathrm{Rh}-\mathrm{Cl}$ frequencies for the type I and II' minima $(\Delta v)$ is $78 \mathrm{~cm}^{-1}$ for $\mathbf{1}^{t \mathbf{B u}}$ and $26 \mathrm{~cm}^{-1}$ for $\mathbf{1}^{\mathbf{P h}}$. On the other hand, the experimental $\mathrm{Rh}-\mathrm{Cl}$ stretching frequency in $\mathbf{1}^{\mathbf{t B u}}$ is only $17 \mathrm{~cm}^{-1}$ lower than that of compound $3^{t \mathbf{B u}}$. Therefore, we conclude that the structure adopted by compound $\mathbf{1}^{t \mathbf{B u}}$ is most likely of type $\mathrm{I}$ in the solid 45 state, like that of compound $\mathbf{3}^{t \mathbf{B u}}$. However, the large chemical shift difference for the $\mathrm{CH}_{2} \mathrm{~S} t \mathrm{Bu} \mathrm{AB}$ resonances in the ${ }^{1} \mathrm{H} \mathrm{NMR}$ spectrum suggests that a structure of type II' might be more favorable in solution for this compound. If this is the case, the difference is certainly a consequence of the lower steric 50 encumbrance of the norbornadiene ligand relative to cyclooctadiene.

The calculated spectra of compounds $\mathbf{1}^{\mathbf{P h}}$ and $\mathbf{3}^{\mathrm{Ph}}$ (shown in the Supporting Information, Figure S3) exhibit similar features, with the major bands in the $260-280 \mathrm{~cm}^{-1}$ range for the type I 55 structures and in the $240-270 \mathrm{~cm}^{-1}$ range for the type II' structures.

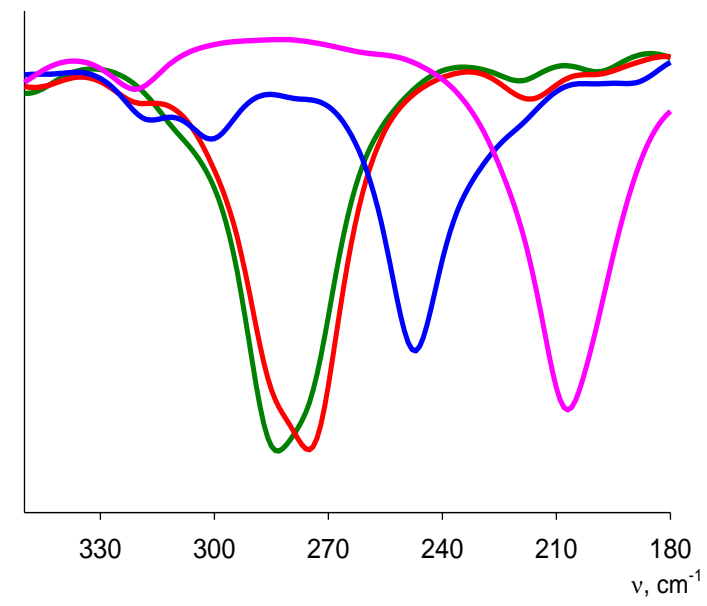

Figure 5. Calculated IR spectra (lower fingerprint region) of $\mathbf{1}^{\mathbf{t B u}}$ type $\mathbf{I}$ (green line), $\mathbf{1}^{\mathrm{Ph}}$ type $\mathbf{I}$ (red line), $\mathbf{1}^{\mathbf{t B u}}$ type II' (pink line) and $\mathbf{1}^{\mathrm{Ph}}$ type II' 60 (blue line).

\section{(d) Catalytic studies}

Because iridium complexes of diphenylphosphinoferrocenyl thioether ligands were shown to be excellent precatalysts for the hydrogenation of acetophenone in the presence of $\mathrm{NaOMe}$ in 65 isopropanol at room temperature under 30 bar of $\mathrm{H}_{2}$ and with only $0.2 \%$ of catalyst, we decided to study the catalytic properties of corresponding rhodium complexes in the same conditions and compare the activity of the complexes (Scheme 4). For these preliminary investigations, we selected only the complexes with 70 t-butyl substituent on sulfur. The results are shown in Table 5.

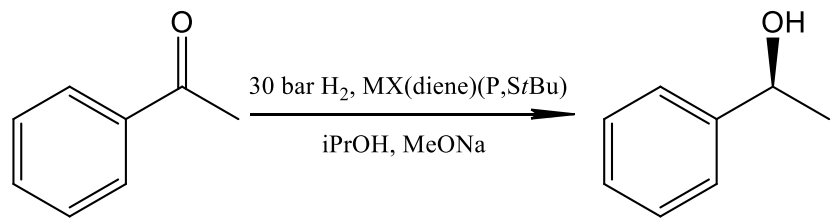

Scheme 4. Asymmetric hydrogenation of acetophenone. 
Table 5. Catalytic activity of the complexes

\begin{tabular}{cccc}
\hline Catalyst & $\begin{array}{c}\text { Time } \\
{[\mathbf{h}]}\end{array}$ & $\begin{array}{c}\text { Conversion } \\
{[\%]}\end{array}$ & ee (S) [\%] \\
\hline (S)- $\mathrm{IrCl}(\mathrm{COD})(\mathrm{P}, \mathrm{S} t \mathrm{Bu})$ & 1 & 93 & 61 \\
$(\mathrm{~S})-\mathrm{RhCl}(\mathrm{COD})(\mathrm{P}, \mathrm{S} t \mathrm{Bu})$ & 1 & 11 & 67 \\
$(\mathrm{~S})-\mathrm{RhCl}(\mathrm{NBD})(\mathrm{P}, \mathrm{S} t \mathrm{Bu})$ & 1 & 12 & 46 \\
$(\mathrm{~S})-\mathrm{Rh}(\mathrm{COD})(\mathrm{P}, \mathrm{S} t \mathrm{Bu}) \mathrm{BF}_{4}$ & 1 & 12 & 51 \\
$(\mathrm{~S})-\mathrm{Rh}(\mathrm{NBD})(\mathrm{P}, \mathrm{S} t \mathrm{Bu}) \mathrm{BF}_{4}$ & 1 & 8 & 49 \\
\hline
\end{tabular}

All the tested rhodium complexes are active precatalysts for 5 the hydrogenation of acetophenone supporting our hypothesis that the rhodium complexes are not only structural but also functional models of the iridium analogues. All these rhodium complexes exhibit a rather similar catalytic activity, which is however significantly lower than that of the corresponding 10 iridium complex $\operatorname{IrCl}(\mathrm{COD})(\mathrm{P}, \mathrm{S} t \mathrm{Bu})$, whereas their enantioselectivities are only slightly lower. A full description of the catalytic performance of these complexes, including the effect of the sulphur substituent, reaction temperature, and substrate, which is currently being investigated, will be reported in due 15 course.

\section{Conclusions}

Rhodium complexes associating chiral ferrocenyl phosphinethioethers and diene (COD or NBD) ligands have been synthesized and fully characterized. For the neutral chlorido 20 complexes, for which X-ray structural analyses could not be carried out, combination of NMR and IR spectroscopy and DFT calculations indicates that they adopt a square planar geometry with a dangling thioether function. A second, pentacoordinated structure with a square pyramidal geometry and the thioether 25 function placed in the axial position is however easily accessible. The cationic complexes, on the other hand adopt a standard square planar bis-chelated structure. These rhodium complexes are not only good structural models for our previously published iridium-based hydrogenation catalysts but have also been tested 30 in the asymmetric hydrogenation of acetophenones and proved to be efficient precatalysts.

\section{Acknowledgement}

We are grateful to the CNRS (Centre National de la RechercheScientifique) and the RFBR (Russian Foundation for ${ }_{35}$ Basic Research) for support through the GDRI (Groupe de RechercheInternationale) "Homogeneous catalysis for Sustainable Development" and a France-Russia (RFBR - CNRS) bilateral grant. We are also grateful to the IUF (InstitutUniversitaire de France) for additional funding and to the ${ }_{40}$ CINES(Centre Informatique National de l'EnseignementSupérieur) and the CICT (Centre Interuniversitaire de Calcul de Toulouse, project CALMIP) for granting free computational time. EMK thanks the Embassy of France in Moscow for a Ph.D. grant.

\section{${ }_{45}$ Notes and references}

${ }^{a}$ CNRS, LCC (Laboratoire de Chimie de Coordination), 205 route de Narbonne, BP 44099, F-31077 Toulouse Cedex 4, France CNRS. Fax:
+33-561553003; Tel: +33-561333174; E-mail: eric.manoury@lcctoulouse.fr.

${ }_{50}{ }^{b}$ Université de Toulouse, UPS, INPT, F-31077 Toulouse Cedex 4, France

${ }^{c}$ A. N. Nesmeyanov Institute of Organoelement Compounds, Russian Academy of Sciences, Vavilov Street 28, 119991 Moscow, Russia; Fax: +7-499-1355085; Tel:+7-499-1356448; E-mail: nataliabelk@ineos.ac.ru.

${ }_{55}{ }^{d}$ Institut Universitaire de France, 103, bd Saint-Michel, 75005 Paris, France.

$†$ Electronic Supplementary Information (ESI) available: [details of any supplementary information available should be included here]. See DOI: $10.1039 / \mathrm{b} 000000 \mathrm{x} /$

60 \$ Footnotes should appear here. These might include comments relevant to but not central to the matter under discussion, limited experimental and spectral data, and crystallographic data.

1. M. Kitamura, T. Ohkuma, S. Inoue, N. Sayo, H. Kumobayashi, S. Akutagawa, T. Ohta, H. Takaya and R. Noyori, J. Am. Chem. Soc., 65 1988, 110, 629-631.

2. T. Ohkuma, H. Ooka, S. Hashiguchi, T. Ikariya and R. Noyori, $J$. Am. Chem. Soc., 1995, 117, 2675-2676.

3. R. Noyori and T. Ohkuma, Angew. Chem. Engl., 2001, 40, 40-73.

4. H. U. Blaser, C. Malan, B. Pugin, F. Spindler, H. Steiner and M.

$70 \quad$ Studer, Advanced Synthesis \& Catalysis, 2003, 345, 103-151.

5. R. Malacea, R. Poli and E. Manoury, Coord. Chem. Rev., 2010, 254, 729-752.

6. W. Tang and X. Zhang, Chemical Reviews (Washington, DC, United States), 2003, 103, 3029-3069.

75 7. T. C. Nugent and M. El-Shazly, Advanced Synthesis \& Catalysis, 2010, 352, 753-819.

8. N. Fleury-Bregeot, V. de la Fuente, S. Castillon and C. Claver, ChemCatChem, 2010, 2, 1346-1371.

9. J. H. Xie, S. F. Zhu and Q. L. Zhou, Chem. Rev., 2011, 111, 1713$80 \quad 1760$

10. D.-S. Wang, Q.-A. Chen, S.-M. Lu and Y.-G. Zhou, Chem. Rev. (Washington, DC, U. S.), 2012, 112, 2557-2590.

11. H.-U. Blaser, Advanced Synthesis \& Catalysis, 2002, 344, 17-31.

12. H. U. Blaser, B. Pugin, F. Spindler and M. Thommen, Acc. Chem. 85 Res., 2007, 40, 1240-1250.

13. G. Iftime, J. Daran, E. Manoury and G. Balavoine, Organometallics, 1996, 15, 4808-4815.

14. G. Iftime, C. MoreauBossuet, E. Manoury and G. Balavoine, Chem. Commun., 1996, 527-528.

90 15. G. Iftime, J. Daran, E. Manoury and G. Balavoine, Angew. Chem., Int. Ed. Eng., 1998, 37, 1698-1701.

16. E. Manoury, J. Fossey, H. Ait-Haddou, J. Daran and G. Balavoine, Organometallics, 2000, 19, 3736-3739.

17. J. Chiffre, Y. Coppel, G. Balavoine, J. Daran and E. Manoury, Organometallics, 2002, 21, 4552-4555.

18. A. M. Masdeu-Bulto, M. Dieguez, E. Martin and M. Gomez, Coord. Chem. Rev., 2003, 242, 159-201.

19. M. Mellah, A. Voituriez and E. Schulz, Chem. Rev., 2007, 107, 5133-5209.

100 20. H. Pellissier, Tetrahedron, 2007, 63, 1297-1330.

21. R. Malacea and E. Manoury, in Phosphorus Ligands in Asymmetric Catalysis, ed. A. Börner, Wiley-VCH, Weinheim, Germany, 2008, vol. 1-3, pp. 749-784.

22. F. L. Lam, F. Y. Kwong and A. S. C. Chan, Chem. Commun., 2010, 46, 4649-4667.

23. L. Routaboul, S. Vincendeau, J.-C. Daran and E. Manoury, Tetrahedron: Asymmetry, 2005, 16, 2685-2690.

24. L. Routaboul, S. Vincendeau, C. O. Turrin, A. M. Caminade, J. P. Majoral, J. C. Daran and E. Manoury, J. Organomet. Chem., 2007, 692, 1064-1073.

25. L. Diab, M. Gouygou, E. Manoury, P. Kalck and M. Urrutigoity, Tetrahedron Lett., 2008, 49, 5186-5189.

26. E. Le Roux, R. Malacea, E. Manoury, R. Poli, L. Gonsalvi and M. Peruzzini, Adv. Synth. \& Catal., 2007, 349, 309-313.

115 27. E. Hauptman, R. Shapiro and W. Marshall, Organometallics, 1998, 
17, 4976-4982.

28. E. Hauptman, P. J. Fagan and W. Marshall, Organometallics, 1999, 18, 2061-2073.

29. S. Gladiali, F. Grepioni, S. Medici, A. Zucca, Z. Berente and L.

$5 \quad$ Kollar, Eur. J. Inorg. Chem., 2003, 556-561.

30. E. Guimet, M. Dieguez, A. Ruiz and C. Claver, Dalton Trans., 2005, 2557-2562.

31. R. Malacea, E. Manoury, L. Routaboul, J.-C. Daran, R. Poli, J. P. Dunne, A. C. Withwood, C. Godard and S. B. Duckett, Eur. J. Inorg. Chem., 2006, 1803-1816.

32. H. J. Drexler, W. Baumann, A. Spannenberg, C. Fischer and D. Heller, J. Organomet. Chem., 2001, 621, 89-102.

33. A. Preetz, H. J. Drexler, C. Fischer, Z. Dai, A. Borner, W. Baumann, A. Spannenberg, R. Thede and D. Heller, Chem. Eur. J., 2008, 14, 1445-1451.

34. A. Preetz, C. Fischer, C. Kohrt, H. J. Drexler, W. Baumann and D. Heller, Organometallics, 2011, 30, 5155-5159.

35. I. D. Gridnev, E. Alberico and S. Gladiali, Chem. Commun., 2012, 48, 2186-2188

20 36. T. Hayashi, T. Mise, M. Fukushima, M. Kagotani, N. Nagashima, Y. Hamada, A. Matsumoto, S. Kawakami, M. Konishi, K. Yamamoto and M. Kumada, Bull. Chem. Soc. Japan, 1980, 53, 1138-1151.

37. A. Altomare, M. Burla, M. Camalli, G. Cascarano, C. Giacovazzo,

25 A. Guagliardi, A. Moliterni, G. Polidori and R. Spagna, J. Appl. Cryst., 1999, 32, 115-119.

38. G. M. Sheldrick, Acta Cryst. A, 2008, 64, 112-122.

39. M. N. Burnett and C. K. Johnson, ORTEPIII, Report ORNL-6895. Oak Ridge National Laboratory, Oak Ridge, Tennessee, U.S. 301996

40. L. J. Farrugia, J. Appl. Crystallogr., 1997, 30, 565.

41. M. J. Frisch, G. W. Trucks, H. B. Schlegel, G. E. Scuseria, M. A. Robb, J. R. Cheeseman, G. Scalmani, V. Barone, B. Mennucci, G. A. Petersson, H. Nakatsuji, M. Caricato, X. Li, H. P. Hratchian, A. F. Izmaylov, J. Bloino, G. Zheng, J. L. Sonnenberg, M. Hada, M. Ehara, K. Toyota, R. Fukuda, J. Hasegawa, M. Ishida, T. Nakajima, Y. Honda, O. Kitao, H. Nakai, T. Vreven, J. Montgomery, J. A., J. E. Peralta, F. Ogliaro, M. Bearpark, J. J. Heyd, E. Brothers, K. N. Kudin, V. N. Staroverov, R. Kobayashi, J.

40 Normand, K. Raghavachari, A. Rendell, J. C. Burant, S. S. Iyengar, J. Tomasi, M. Cossi, N. Rega, N. J. Millam, M. Klene, J. E. Knox, J. B. Cross, V. Bakken, C. Adamo, J. Jaramillo, R. Gomperts, R. E. Stratmann, O. Yazyev, A. J. Austin, R. Cammi, C. Pomelli, J. W. Ochterski, R. L. Martin, K. Morokuma, V. G. Zakrzewski, G. A. 45 Voth, P. Salvador, J. J. Dannenberg, S. Dapprich, A. D. Daniels, Ö. Farkas, J. B. Foresman, J. V. Ortiz, J. Cioslowski and D. J. Fox, Gaussian 09, Revision A.02, Gaussian, Inc., Wallingford CT, 2009.

42. A. D. Becke, J. Chem. Phys., 1993, 98, 5648-5652.

43. C. T. Lee, W. T. Yang and R. G. Parr, Phys. Rev. B, 1988, 37, 785$50 \quad 789$.

44. D. Andrae, U. Haussermann, M. Dolg, H. Stoll and H. Preuss, Theor. Chim. Acta, 1990, 77, 123-141.

45. U. Haussermann, M. Dolg, H. Stoll, H. Preuss, P. Schwerdtfeger and R. M. Pitzer, Mol. Phys., 1993, 78, 1211-1224.

55 46. W. Kuchle, M. Dolg, H. Stoll and H. Preuss, J. Chem. Phys., 1994, 100, 7535-7542.

47. T. Leininger, A. Nicklass, H. Stoll, M. Dolg and P. Schwerdtfeger, J. Chem. Phys., 1996, 105, 1052-1059.

48. A. W. Ehlers, M. Bohme, S. Dapprich, A. Gobbi, A. Hollwarth, V.
Jonas, K. F. Kohler, R. Stegmann, A. Veldkamp and G. Frenking, Chem. Phys. Lett., 1993, 208, 111-114.

49. R. Malacea, E. Manoury, J.-C. Daran and R. Poli, J. Mol. Struct., 2008, 890, 249-254.

50. R. Malacea, L. Routaboul, E. Manoury, J.-C. Daran and R. Poli, J. 65 Organomet. Chem, 2008, 693, 1469-1477.

51. L. Diab, J. C. Daran, M. Gouygou, E. Manoury and M. Urrutigoity, Acta Cryst. C, 2007, 63, M586-M588.

52. F. Teixidor, R. Benakki, C. Vinas, R. Kivekas and R. Sillanpaa, Organometallics, 1998, 17, 4630-4633.

$7053 . \quad$ D. A. Evans, F. E. Michael, J. S. Tedrow and K. R. Campos, J. Am. Chem. Soc., 2003, 125, 3534-3543.

54. N. Khiar, R. Navas, E. Alvarez and I. Fernandez, Arkivoc, 2008 , 211-224.

55. N. Khiar, R. Navas, B. Suarez, E. Alvarez and I. Fernandez, Org. Lett., 2008, 10, 3697-3700.

56. J. Browning, G. W. Bushnell, K. R. Dixon and R. W. Hilts, $J$. Organomet. Chem., 1993, 452, 205-218.

57. J. W. Faller, S. C. Milheiro and J. Parr, J. Organomet. Chem., 2008, 693, 1478-1493.

80 58. J. Browning, K. R. Dixon, N. J. Meanwell and F. Wang, $J$. Organomet. Chem., 1993, 460, 117-126.

59. H. S. Lee, J. Y. Bae, D. H. Kim, H. S. Kim, S. J. Kim, S. Cho, J. Ko and S. O. Kang, Organometallics, 2002, 21, 210-219.

60. A. Neveling, G. R. Julius, S. Cronje, C. Esterhuysen and H. G. 85 Raubenheimer, Dalton Trans., 2005, 181-192.

61. J. A. Mata, A. R. Chianese, J. R. Miecznikowski, M. Poyatos, E. Peris, J. W. Faller and R. H. Crabtree, Organometallics, 2004, 23, 1253-1263.

62. M. V. Baker, S. K. Brayshaw, B. W. Skelton, A. H. White and C. 90 C. Williams, J. Organomet. Chem., 2005, 690, 2312-2322.

63. R. J. Lowry, M. K. Veige, O. Clement, K. A. Abboud, I. Ghiviriga and A. S. Veige, Organometallics, 2008, 27, 5184-5195.

64. J. J. Robertson, A. Kadziola, R. A. Krause and S. Larsen, Inorg. Chem., 1989, 28, 2097-2102.

95 65. M. Toganoh, N. Harada and H. Furuta, J. Organomet. Chem., 2008, 693, 3141-3150.

66. H. F. Haarman, F. R. Bregman, J. M. Ernsting, N. Veldman, A. L. Spek and K. Vrieze, Organometallics, 1997, 16, 54-67.

67. L. H. Gade, V. César and S. Bellemin-Laponnaz, Angew. Chem 100 Engl., 2004, 43, 1014-1017.

68. V. César, S. Bellemin-Laponnaz and L. H. Gade, Eur. J. Inorg. Chem., 2004, 3436-3444.

69. V. César, S. Bellemin-Laponnaz, H. Wadepohl and L. H. Gade, Chem. Eur. J., 2005, 11, 2862-2873.

105 70. M. Stradiotto, J. Cipot and R. McDonald, J. Am. Chem. Soc., 2003 , 125, 5618-5619.

71. A. D. Phillips, S. Bolaño, S. S. Bosquain, J.-C. Daran, R. Malacea, M. Peruzzini, R. Poli and L. Gonsalvi, Organometallics, 2006, 25, 2189-2200.

110 72. M. Ahlmann and O. Walter, J. Organomet. Chem., 2004, 689, 3117-3131.

73. A. M. Z. Slawin, M. B. Smith and J. D. Woollins, J. Chem. Soc., Dalton Trans., 1996, 1283-1293.

74. A. M. Z. Slawin, M. B. Smith and J. D. Woollins, J. Chem. Soc., 115 Dalton Trans., 1996, 4575-4581. 